\title{
Multi-scale Deep Nearest Neighbors
}

by

\author{
Abhijeet Chauhan \\ A Thesis submitted to \\ the Faculty of Graduate Studies and Research \\ in partial fulfilment of \\ the requirements for the degree of \\ Master of Computer Science \\ Department of Computer Science \\ Carleton University \\ Ottawa, Ontario, Canada
}

September 2020

Copyright (c)

2020 - Abhijeet Chauhan 
The undersigned recommend to

the Faculty of Graduate Studies and Research

acceptance of the Thesis

\title{
Multi-scale Deep Nearest Neighbors
}

\author{
Submitted by Abhijeet Chauhan \\ in partial fulfilment of the requirements for the degree of \\ Master of Computer Science
}

Dr. Majid Komeili, Thesis Supervisor

Dr. Matthew Holden, Internal Examiner

Dr. Paula Branco, External Examiner

Dr. Oliver van Kaick, Department Chair

Carleton University

2020 


\section{Abstract}

The performance of the k-NN classifier is dependent on its input space. The predictive power of the $\mathrm{k}-\mathrm{NN}$ classifier decreases in high dimensional spaces due to the curse of dimensionality where all pairs of samples have almost the same distance. $\mathrm{k}-\mathrm{NN}$ has been used in a variety of applications in different areas such as computer vision, natural language processing, and recommender systems. Many approaches in the literature try to improve the input space of k-NN. These approaches include manually extracting a low dimensional feature space, or learning a mapping into a low-dimensional space. The mapping of the input space to a low-dimensional space is learned through linear or non-linear transformation.

In this thesis, we aim to learn a deep embedding space suitable for k-NN. Our approach is based on minimizing the leave-one-out 1-NN classification error in the embedding space. That is, if we hold out a training sample, it should be correctly classified using 1-NN applied to the rest of the training data. Directly optimizing for such a rule is not tractable due to its discontinuous nature. We propose Multi-scale Deep Nearest Neighbor (MsDNN) which is a differentiable loss function that aims to maximize the expected sample margin for every training sample.

The output of MsDNN is an embedding space. We evaluate the resulting space from two angles. From the classification view, during testing, we run a k-NN classifier and report the classification accuracy. But classification accuracy does not tell us 
the entire story about the goodness of an embedding space. Therefore, we run kmeans clustering in the embedding space. We hypothesize that if a class consists of several disjoint sets (also known as subclasses or clusters), a good embedding space should keep them separate. So, a simple clustering method such as k-means would identify them and yield high clustering accuracy. Analogous to hierarchical clustering, subclasses might exist on different scales. Our method provides a mechanism to target subclasses in different scales. We empirically demonstrate that the embedding space learned by MsDNN can preserve the relationship between the data to discover subclasses while separating classes that were originally given during training. 


\section{Acknowledgments}

I would like to thank my supervisor, Professor Majid Komeili, for providing a supportive environment and invaluable feedback. Without his guidance and knowledge in machine learning, this thesis would not have been possible. I am grateful to my supervisor for encouraging me whenever I got stressed and worried. I would like to acknowledge the support of Carleton University and its technical staff. I would like to thank my friends and family for always believing in me.

Overall, I am grateful for this great journey and an amazing experience. 


\section{Table of Contents}

Abstract $\quad$ iii

Acknowledgments $\quad$ V

Table of Contents $\quad$ vi

List of Tables $\quad$ ix

List of Figures $\quad$ X

1 Introduction 1

1.1 What is the problem? . . . . . . . . . . . . . 1

1.2 Why is this problem important? . . . . . . . . . . . 1

1.3 What is already done in the literature? . . . . . . . . . . . 2

1.4 What is not addressed? . . . . . . . . . . . . . . . . . 3

1.5 Contributions ......................... 4

1.6 Organization of Thesis . . . . . . . . . . . . . 4

2 Background $\quad 6$

2.1 Introduction . . . . . . . . . . . . . . . 6

2.2 K Nearest Neighbors . . . . . . . . . . . . . . . . . 6

2.2 .1 Algorithm .................... 7

2.2.2 Supervised Metric Learning . . . . . . . . . . . . . . . . 9 
2.3 Deep Learning . . . . . . . . . . . . . . . . . . . . . . . . . . . . . . . 12

2.3.1 Relation with Neurons in the Brain . . . . . . . . . . . . 13

2.3 .2 Feedforward Deep Network . . . . . . . . . . . . . . . . . 15

2.3 .3 Loss Function . . . . . . . . . . . . . . . . . . . . . . . . 18

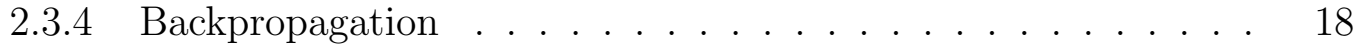

$2.3 .5 \quad$ Optimizers . . . . . . . . . . . . . . . . . . . . 19

2.4 Convolutional Neural Networks . . . . . . . . . . . . . . . . . 23

2.4 .1 Convolution Layer . . . . . . . . . . . . . . . . . . . 24

2.4 .2 Pooling Layer . . . . . . . . . . . . . . . . . 26

2.4 .3 Properties . . . . . . . . . . . . . . . . 27

2.4.4 Popular Architectures . . . . . . . . . . . . . . . . . . 29

3 Related Work $\quad 30$

3.1 Deep Extensions of Nearest Neighbors _... . . . . . . . . . 30

3.2 Deep Metric Learning . . . . . . . . . . . . . . . . . . . . . . . . 33

3.2 .1 Contrastive Loss _ . . . . . . . . . . . . . . . . . 33

3.2 .2 Triplet Loss . . . . . . . . . . . . . . . . . . . 35

3.2 .3 Other methods inspired by Triplet loss . . . . . . . . . . 37

4 Proposed Method $\quad 39$

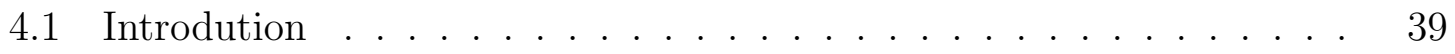

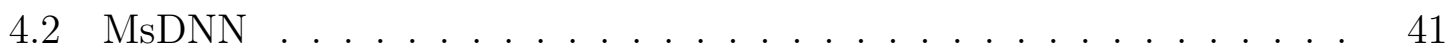

4.3 Summary . . . . . . . . . . . . . . . . . . 44

5 Experimental Results $\quad 45$

5.1 Datasets . . . . . . . . . . . . . . . . . . 45

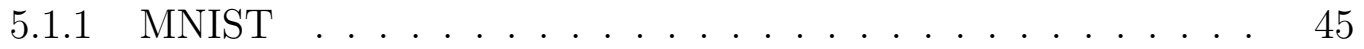

5.1 .2 Fashion MNIST . . . . . . . . . . . . . . . 46 
$5.1 .3 \quad$ USPS . . . . . . . . . . . . . . . . . . . 47

5.1 .420 Newsgroup . . . . . . . . . . . . . . . . . . . . 48

5.2 Evaluation Metrics . . . . . . . . . . . . . . . . 49

5.2.1 Evaluating clustering performance. . . . . . . . . . 49

5.2.2 Evaluating classification performance . . . . . . . . . . . 51

$5.3 \mathrm{MsDNN}$ for learning at different scales . . . . . . . . . . . . 52

5.4 Evaluating the embedding space . . . . . . . . . . . . . 57

5.5 Computational complexity . . . . . . . . . . . . . . 61

5.6 Implementation details . . . . . . . . . . . . . . . . . 61

5.6.1 Model Selection . . . . . . . . . . . . . . . 64

5.7 Limitations . . . . . . . . . . . . . . . . . . 65

5.8 Summary . . . . . . . . . . . . . . . . 66

$\begin{array}{lll}6 & \text { Conclusions } & 67\end{array}$

$\begin{array}{lll}7 & \text { Future Works } & 69\end{array}$

$\begin{array}{ll}\text { List of References } & 70\end{array}$ 


\section{List of Tables}

2.1 Activation Functions . . . . . . . . . . . . . . . . . 15

5.120 newsgroup topic grouped together based on the subject matter . . 48

5.2 Evaluation of the proposed method on the train set. $\left(^{\dagger}\right),\left({ }^{\S}\right),\left(^{\ddagger}\right),\left(^{\dagger \dagger}\right)$ denotes that the results are reported in [66], [67], [68], and [69] respec-

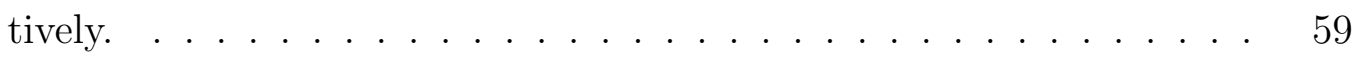

5.3 Evaluation of the classification accuracy of the proposed method on the test set. . . . . . . . . . . . . . . 60 


\section{List of Figures}

2.1 (a) shows the dataset where triangle and square are different classes and (b) demonstrates the behaviour of k-NN when $\mathrm{K}$ is 3. Solid dot is the test sample and circle shows the K nearest samples. . . . . . . . 7

2.2 Biological Neuron . . . . . . . . . . . . . . . . . . . . . . . . . . . 13

2.3 Artificial Neuron . . . . . . . . . . . . . . . . . . . . . . . . . . 14

2.4 Deep Feedforward Networks [19]. . . . . . . . . . . . . . . . 16

2.5 Sigmoid Function $\ldots \ldots \ldots \ldots \ldots \ldots \ldots \ldots$

2.6 The green point represents the current position for loss and weight. Partial derivative is computed at this point. (a) shows negative partial derivative and (b) shows positive partial derivative . . . . . . 19

2.7 The loss can get stuck in local optima with gradient descent $\ldots . .20$

2.8 The momentum manages to cross the local optima and move toward global optima. . . . . . . . . . . . . . . . . . . . . . . . 21

2.9 General layers in a Convolutional Neural Network. . . . . . . . . . . 24

2.10 Convolution operation $\ldots \ldots \ldots \ldots \ldots \ldots$

2.11 Padded input at beginning with length equal to $P_{\text {start }}$ shown in red and at the end with length equal to $P_{\text {end }}$ shown in blue. . . . . 26

2.12 Max Pooling . . . . . . . . . . . . . . . . . 27

2.13 (a) is a regular neural network. (b) is a convolutional neural network with kernel width of $3[19] \ldots \ldots \ldots \ldots \ldots$ 
$3.1 \quad N^{3}$ block . . . . . . . . . . . . . . . . . . . 32

3.2 Contrastive loss looks at absolute distance and Triplet Loss looks at relative distance . . . . . . . . . . . . . . . . . . . . . . . 34

3.3 Semi-Hard Triplet Mining . . . . . . . . . . . . . . . . . 36

5.1 Examples from the MNIST dataset. . . . . . . . . . . . . . 46

5.2 Examples from the FMNIST dataset. . . . . . . . . . . . . 47

5.3 Examples from the USPS dataset [52]. . . . . . . . . . . . . . 47

5.4 Effect of minimizing the MsDNN loss by applying gradient descent on the input space. (a) 2-dimensional synthetic data with two classes shown in yellow and purple. Each class initially has two subclasses. (b) The resulting input space when $\sigma$ is small. (c) The resulting input space when $\sigma$ is large.

5.5 t-SNE plots for the MNIST dataset. (a) When $\sigma$ is small. Colors show the two classes. (b) The same as (a) but colors show the ground-truth values for the 10 subclasses. (c) When $\sigma$ is large. Colors show the two classes. (d) The same as (c) but colors show the ground-truth values the 10 subclasses.

5.6 Pairwise symmetric KL-divergence between subclasses. (a) for the MNIST dataset when $\sigma$ is small. (b) for the MNIST dataset when $\sigma$ is large. (c) for the USPS dataset when $\sigma$ is small. (d) for the USPS dataset when $\sigma$ is large. Lighter colors denote lower KL-divergence. . . . . . . . . . . . .

5.7 NMI for different values of $\sigma$. (a) MNIST. (b) USPS. (c) 20NewsGroup. (d) F-MNIST. . . . . . . . . . . . . . . . 56

5.8 Network Architecture for MNIST and Fashion MNIST. . . . . . . . 63

5.9 Network Architecture for USPS . . . . . . . . . . . . . . . . 64

5.10 Network Architecture for 20Newsgroup . . . . . . . . . . . 65 


\section{Chapter 1}

\section{Introduction}

K nearest neighbor $(\mathrm{k}-\mathrm{NN})[1]$ is an algorithm that predicts the output of test data by looking at the outputs of the $\mathrm{k}$ nearest samples in the training set. A distance metric such as euclidean is used to find the $\mathrm{k}$ nearest samples from the test data.

\subsection{What is the problem?}

The performance of the $\mathrm{k}-\mathrm{NN}$ degrades in high dimensional space due to the curse of dimensionality [2]. In high dimensional space, the distance between pairs of samples is almost the same which means the test data have almost the same distance from the nearest and the farthest sample. It is essential to reduce the dimension of input space to improve the predictive power of k-NN.

\subsection{Why is this problem important?}

k-NN does not make any assumption about the underlying distribution of data. This is very useful in the tasks where we have little or no prior knowledge of the distribution

of the data. K-nearest neighbor is one of the most intuitive and interpretable classifiers in that the intuition behind the model is easy to understand; a decision can be 
understood by its neighboring samples. These properties make k-NN suitable for a variety of applications. In [3], a feature space is learned by dimension reduction followed by k-NN for face recognition. Smile [4] and hand gesture [5] recognition are some other applications of k-NN in computer vision. The k-NN has been applied to text mining for the categorization and retrieval of most relevant documents [6]. In healthcare, the algorithm is used for monitoring [7] and diagnosis [8] purposes. $\mathrm{k}-\mathrm{NN}$ is also used in recommender systems [9] to improve the accuracy of the recommendation.

\subsection{What is already done in the literature?}

The performance of k-NN is dependent on the quality of the input space it is applied to. Thus, there have been many approaches in the literature to find feature space from the input data which improves the predictive power of k-NN. Traditionally, feature descriptors are applied to learn a low dimensional representation of the input image to keep the most important information. In [10], the histogram of gradients is computed in different orientations (HOG) [11] to extract features from the input image. $\mathrm{k}-\mathrm{NN}$ is applied to this feature space to recognize hand gestures. Another feature descriptor to extract features from an input image is by using Local Binary Patterns (LBP) [12] that focuses on extracting local image texture. These features are then fed to k-NN in [13] for automatic age classification. For text categorization,

in [14], features are extracted using TF-IDF [15] which is influenced by the frequency of a word in the document and the number of documents that contain the word and $\mathrm{k}-\mathrm{NN}$ on this feature space is used to categorize text.

Rather than manually extracting features from input data, one can learn a representation that projects the input to an embedding space tailored for k-NN. Neighborhood component analysis (NCA) [16] learns a linear transformation to maximize the leave-one-out classification performance in the transformed space. In [16], NCA 
is used to project the input to a lower-dimensional space where $\mathrm{k}-\mathrm{NN}$ is used to perform handwritten digit recognition. In large margin nearest neighbor (LMNN) [17], a linear transformation is learned where for a test data, the k nearest neighbors are encouraged to be of the same class and the samples from other classes are pushed away. This transformation is combined with $\mathrm{k}-\mathrm{NN}$ for applications such as face recognition, letter recognition, text categorization, and handwritten digit recognition [18].

More recently in the field of deep learning [19], deep neural networks have been used to learn a non-linear transformation into an Embedding space where the samples from different classes are pushed away while samples from the same class nearby are pulled together [20]. To learn such a projection of input to a latent space many methods are motivated by various loss functions that tune the neural network weights to achieve the stated low dimensional latent space. Contrastive loss [21] considers pairs of samples at a time and the loss function minimizes the distance between samples from the same class and maximizes the distance between samples from different classes. Instead of absolute distance, triplet loss [22] considers the relative distance of examples to an anchor. In this approach, a triplet, anchor, positive, and negative, is considered at a time. The positive has the same label as that of anchor whereas negative has a label different from the anchor. The loss is motivated to increase the distance between the anchor and hardest negative (i.e. the nearest negative) while decreasing the distance between the anchor and hardest positive (i.e. farthest positive). There are many approaches such as N pair loss [23], anchor loss [24], and mixed loss [25] that are inspired by triplet loss.

\subsection{What is not addressed?}

The triplet loss is a discontinuous function and shows poor convergence behaviour [3] and often additional steps for triplet selection [3] or constructing batches [26] are 
required to make a neural network converge with triplet loss. Another issue is that it assumes that classes have a single mode. It does not allow disjoint sets because it pulls the farthest positive towards the anchor. In many applications, it is essential to learn an embedding space that preserves the subclass or clusters within the classes originally fed to the classifier.

\subsection{Contributions}

We propose multi-scale deep nearest neighbors (MsDNN), a novel loss function to learn an embedding space that aims to satisfy the above shortcomings. In particular, MsDNN allows disjoint sets. MsDNN allows feature representation learning with coarse labels while subclasses can remain separated. Also, MsDNN is a differentiable loss function for training a neural network in an end-to-end fashion, eliminating the need for additional treatments such as a carefully designed triplet selection [3] or batches construction [26] mechanism mentioned above.

We perform experiments on four different datasets to empirically demonstrate the effectiveness of MsDNN in learning embedding spaces that reveal unknown sub-classes at different scales. We empirically demonstrate that the embedding space learned by MsDNN can preserve subclasses that can be identified by common clustering methods. This is in contrast to the mainstream of the previous works that assume classes have a single mode. Analogous to the hierarchical clustering, MsDNN can be used to target clusters at different scales.

\subsection{Organization of Thesis}

The thesis is organized into seven chapters. This is the first chapter for the introduction which provides motivation, contribution, and organization of the thesis. 
Details for other chapters are as follows:-

Chapter 2 provides the background knowledge for the thesis which covers $\mathrm{K}$ Nearest Neighbors, Deep Learning, and Convolutional Neural Networks.

Chapter 3 describes the related work done in the literature and discusses what they have done and their shortcomings.

Chapter 4 gives details about the method proposed providing intuition and mathematical equations to understand the working of MsDNN.

Chapter 5 consists of multiple experiments done to examine different aspects of the proposed method. We will evaluate and compare MsDNN with related methods.

Chapter 6 concludes the thesis.

Chapter 7 gives some ideas for the future direction of the research. 


\section{Chapter 2}

\section{Background}

\section{$2.1 \quad$ Introduction}

Our proposed method learns a representation of the data using neural networks [27] that is suitable for K nearest neighbor classifier. In this section, we cover the essential building blocks for our method. These blocks are K Nearest Neighbors [1] and Deep Learning [19].

\subsection{K Nearest Neighbors}

In machine learning [28], which is a field of computer science that learns patterns from the raw data, the $\mathrm{k}$ nearest neighbor $(\mathrm{k}-\mathrm{NN})[1]$ is a method that infers the label of test data by looking at the labels (supervisory signal) of the nearby samples in the train set. Since we have an output label during training, k-NN is a supervised machine learning method. When the desired output label is discrete we learn to perform classification task and when the desired output label is continuous the task is called regression. k-NN is suitable for both classification and regression tasks. The algorithm is described in detail with an example in the next section. 


\subsubsection{Algorithm}

k-NN method, for a test sample, finds $\mathrm{k}$ nearest samples and predicts the label typically through majority voting for classification task and label is predicted by taking the average for regression task. The following example is to explain the concept of $\mathrm{k}$-NN. The figure shows data residing in 2-dimensional space. In this example, we have two classes represented by square and triangle in Figure 2.1.

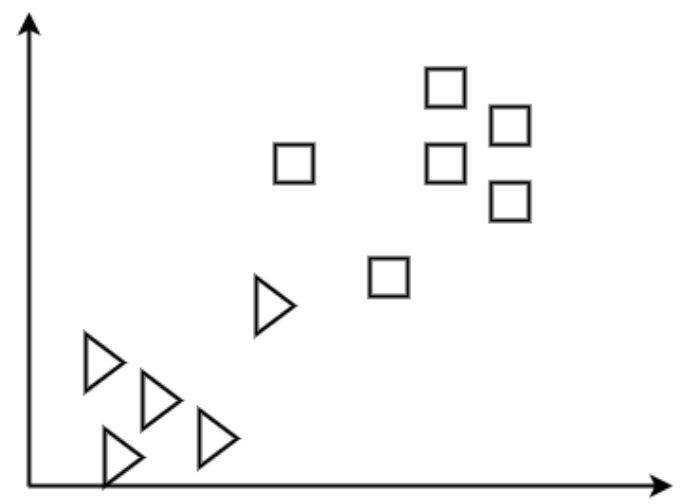

(a)

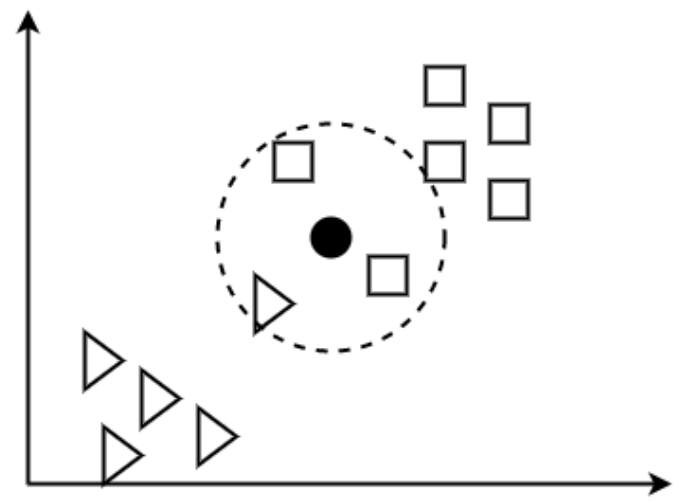

(b)

Figure 2.1: (a) shows the dataset where triangle and square are different classes and (b) demonstrates the behaviour of $\mathrm{k}-\mathrm{NN}$ when $\mathrm{K}$ is 3 . Solid dot is the test sample and circle shows the $\mathrm{K}$ nearest samples.

For our example, we set the value of $\mathrm{K}$ to be 3 . The choice of value for $\mathrm{K}$ is dependent on the data. Generally, the higher $\mathrm{K}$ helps to reduce noise and variance of the predictions. A good K can be selected by doing a grid search on various values of $\mathrm{K}$ and choosing the one with the best performance. For test data, like the solid circle in our example, $\mathrm{k}-\mathrm{NN}$ calculates the distance between test data and every training data. The distance can be Euclidean, manhattan, etc. We choose Euclidean in our example and apply the Equation 2.1 on 2-dimensional inputs $\mathrm{x}$ and $\mathrm{y}$.

$$
d_{\text {Euclidean }}=\sqrt{\left(x_{1}-y_{1}\right)^{2}+\left(x_{2}-y_{2}\right)^{2}}
$$

Next, the distances computed are sorted in ascending order, and the first $\mathrm{K}$ 
distances are selected. Finally, the test point is assigned to the class based on majority voting. In our case, the test class is assigned to the square class.

One simple extension of k-NN is called weighted nearest neighbor where the nearest neighbor is assigned more weight than the more distant ones. This can be achieved by giving the nearest $\mathrm{k}$ neighbors the weight of $1 / \mathrm{d}$ where $\mathrm{d}$ is the distance from the test sample, also known as an inverse distance function. The distance function used is also called a kernel function. The prediction under this scheme uses the following formulas:

For regression,

$$
y=\frac{\sum_{i=1}^{k} w_{i} y_{i}}{\sum_{i=1}^{k} w_{i}}
$$

where, $w_{i}$ is the weight for input $x_{i}$ with label $y_{i}$. For classification,

$$
y=\operatorname{argmax}_{v} \sum_{i=1}^{k} w_{i} * I\left(v=y_{i}\right)
$$

where, $v$ represents the set of labels and similar to regression, $w_{i}$ is the weight for input $x_{i}$ with label $y_{i} . \mathrm{I}($.$) is an indicator function that returns 1$ when the equality inside is true and 0 when the equality is false. The argmax makes sure to assign the prediction label that has the highest value.

When we consider a weighted nearest neighbor, where all the nearest $\mathrm{K}$ neighbors have equal weights, the weighted nearest neighbor classifier is equivalent to $\mathrm{k}-\mathrm{NN}$. In other words, for $\mathrm{k}-\mathrm{NN}$ the distance or kernel function used is $1 / \mathrm{k}$, where $\mathrm{k}$ is the number of nearest samples considered for prediction.

$\mathrm{k}-\mathrm{NN}$ algorithm is independent of the distribution of the data. It is intuitive to 
find an outlier with k-NN by finding an example of a class that is surrounded by labels from other classes. k-NN provides interpretable prediction because the choice of class can be explained by the nearest samples.

When the Euclidean distance is used to compute the distance between two points in high dimensional space, k-NN suffers from the curse of dimensionality. The dimension reduction algorithm helps to improve the performance of k-NN classification. $\mathrm{k}-\mathrm{NN}$ is very sensitive to noisy samples which can corrupt the predictions. The performance can be highly improved if we learn a representation of data that is less sensitive to noisy samples. In the next section, we discuss ways to improve k-NN classifier. Another drawback of $\mathrm{k}-\mathrm{NN}$ is the fact that all the training data has to be stored in the memory and inference becomes slower with an increase in data.

\subsubsection{Supervised Metric Learning}

The purpose of metric learning [29] is to learn a distance metric where the distance between samples from the same classes is small and the distance between samples from different classes is large. In the following we will discuss two representative works for learning a metric to improve the classification performance of k-NN.

\section{Large Margin Nearest Neighbor}

Large Margin Nearest Neighbor [17] is an algorithm to learn a distance metric where all the data points in the training set are surrounded by $\mathrm{k}$ samples from the same class. This algorithm is performed to minimize leave-one-out error. The algorithm learns a Mahalanobis distance metric that is mathematically defined as,

$$
d\left(x_{i}, x_{j}\right)=\left(x_{i}-x_{j}\right)^{T} M\left(x_{i}-x_{j}\right)
$$


where $x_{i}$ and $x_{j}$ are pairs of samples from the same class. $M$ is a positive semi-definite matrix. When M is identity, the Mahalanobis distance is equal to Euclidean distance.

For every data point $x_{i}$, there are two types of data points :

- Target neighbors: These are the labels that become the nearest neighbor by using the learned matrix $M$.

- Imposter: These are the samples from other class

LMNN aims to minimize the distance between $x_{i}$ and the target label $x_{j}$ and maximize the distance between $x_{i}$ and the imposter $x_{l}$. The first objective is represented mathematically as follow:

$$
\sum_{i, j \in N_{i}} d\left(x_{i}, x_{j}\right)
$$

where, $N_{i}$ is the set of target neighbors for a data point $x_{i}$. The second goal is the maximize the distance between $x_{i}$ and the imposter that are less than one unit away relative to the target neighbors.

$$
E_{i j l}=\sum_{i, j \in N, l, y_{l} \neq y_{i}}\left[d\left(x_{i}, x_{j}\right)+1-d\left(x_{i}, x_{l}\right)\right]_{+}
$$

where $x_{l}$ is the imposter and $[\cdot]_{+}=\max (x, 0)$ is the hinge operator. The overall optimization is defined as,

$$
\min _{M} \sum_{i, j \in N} d\left(x_{i}, x_{j}\right)+\lambda \sum_{i, j, l} E_{i j l}
$$


with constraints,

$$
\begin{array}{r}
\forall_{i, j \in N_{i}, l, y_{l} \neq y_{i}} \\
d\left(x_{i}, x_{j}\right)+1-d\left(x_{i}, x_{l}\right) \leq E_{i j l} \\
E_{i j l} \geq 0 \\
M \geq 0
\end{array}
$$

The objective function combines the two goals. $\lambda$ is a hyperparameter that controls the influence of the second goal and $M \geq 0$ constraint makes sure that $\mathrm{M}$ is positive semi-definite.

\section{Neighborhood Component Analysis}

Neighborhood component analysis (NCA) [16] aims to find a linear transformation to maximize the LOO classification performance in the transformed space. NCA considers stochastic ("soft") nearest neighbor in the transformed space. These neigh-

bors are defined using softmax over Euclidean distance in the transformed space. Mathematically,

$$
p_{i j}=\frac{\exp \left(-\left\|A x_{i}-A x_{j}\right\|^{2}\right)}{\sum_{k} \exp \left(-\left\|A x_{i}-A x_{k}\right\|^{2}\right)}
$$

where $j \neq i$ and when $j=i, p_{i j}=0$.

Equation 2.8 computes the probability of $x_{j}$ being neighbor of the point $x_{i}$ after linear transformation A. Since this assignment of neighborhood is not discrete, it is called soft nearest neighbor. Using the properties of probability theory, the probabiliy of correctly classifying a point $x_{i}$ is defined as, 


$$
p_{i}=\sum_{j \in C_{i}} p_{i j}
$$

where $C_{i}$ is the set of points with same class label.

NCA's goal is to maximize the expected number of points correctly classified using the following equation,

$$
f(A)=\sum_{i} \sum_{j \in C_{i}} p_{i j}=\sum_{i} p_{i}
$$

This objective function is differentiable and thus can easily be learned with stochastic gradient descent.

Since our work is in the intersection of k-NN and deep neural networks, in the following, we will provide some background information on deep learning, in particular the network structures that we will use later on in our experiments.

\subsection{Deep Learning}

Deep Learning is an approach to AI. One of the traditional approaches to AI is to use formal language and define rules and relations. This approach works for the type of intelligence when humans have an understanding of these rules and relations. However, for things that are learned from experience and we don't know the exact rules this approach fails. For these intuitive problems like recognizing a face or detecting an object, a deep learning approach is to learn these tasks from experience. From experience, we learn knowledge by learning concepts directly from the raw data. This approach doesn't require humans to provide any knowledge in terms of rules and relations.

AI systems need the ability to acquire knowledge by extracting patterns from the raw data. The performance of these systems is dependent on the representation of the data that is fed. One way to define these representations is to extract features for 
that task. However, for complex tasks finding the right set of features is very difficult. Deep learning solves this problem by learning representations from data.

\subsubsection{Relation with Neurons in the Brain}

Building blocks of deep learning are neural networks that are composed of units called neurons. These neurons are organized into layers to form a network. In this section, we will cover neurons and draw similarities between neurons in a neural network and neuron in the brain. Figure 2.2, shows an illustration of a typical neuron in brain. The biological neuron collects electrical signals from dendrites and axon is the authority that decides when to fire up an electrical signal that acts as an output.

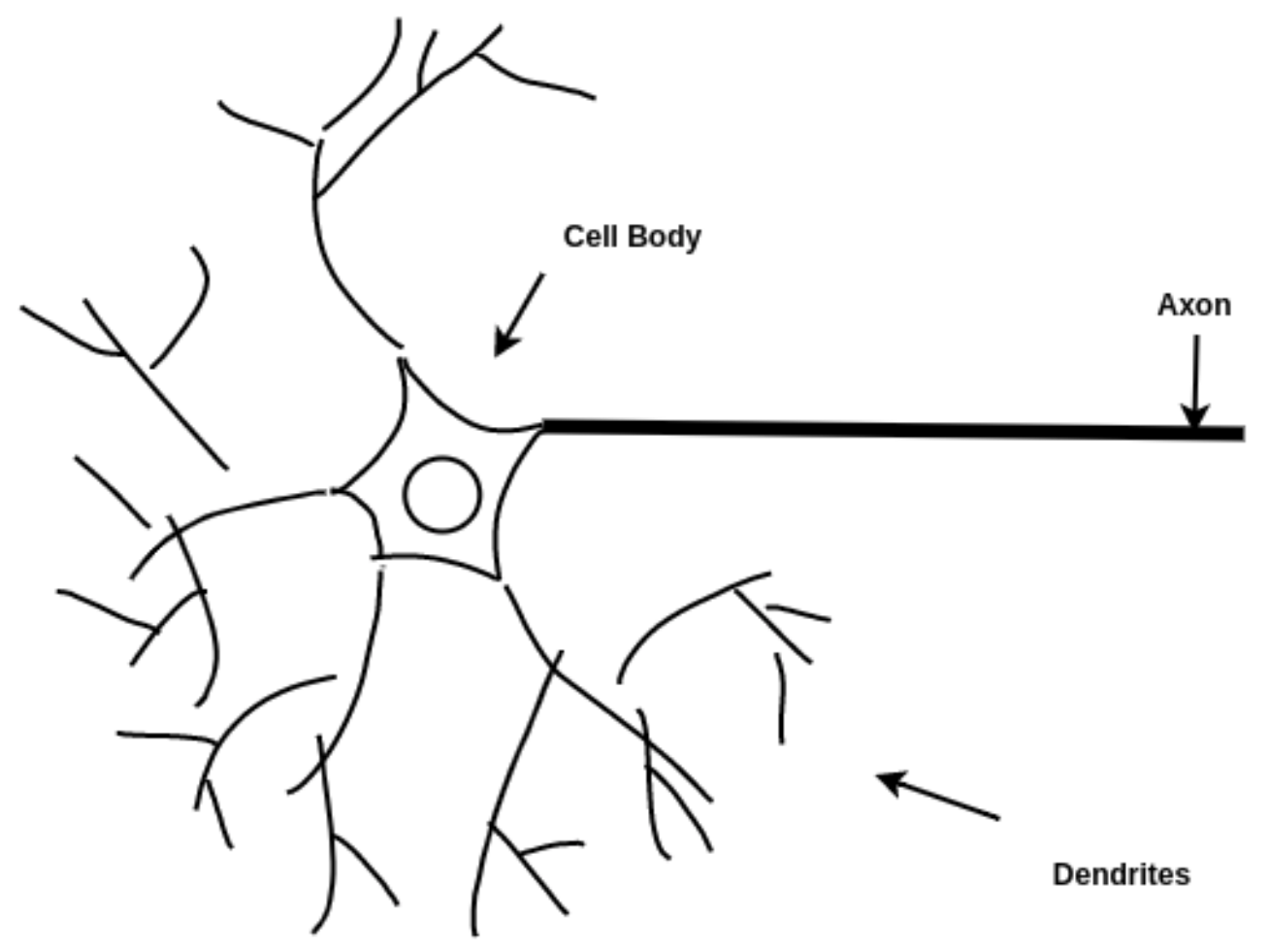

Figure 2.2: Biological Neuron

Figure 2.3 demonstrates artificial neurons that are used in deep learning to build neural networks. Similar to biological neural networks, artifical neural networks take 
inputs and the value of the output depends on the weight and bias which can be trained. Mathematically, the equations for output is as follows:

$$
O U T P U T=F\left(\sum_{i=1}^{N} x_{i} w_{i}+\text { Bias }\right)
$$

where $\mathrm{N}$ is the number of inputs and $\mathrm{F}$ is an activation function. The weighted sum of inputs can be written as a dot product between the weights and inputs which is preferred for GPU.

$$
\sum_{i=1}^{N} x_{i} * w_{i}=x^{T} * w
$$

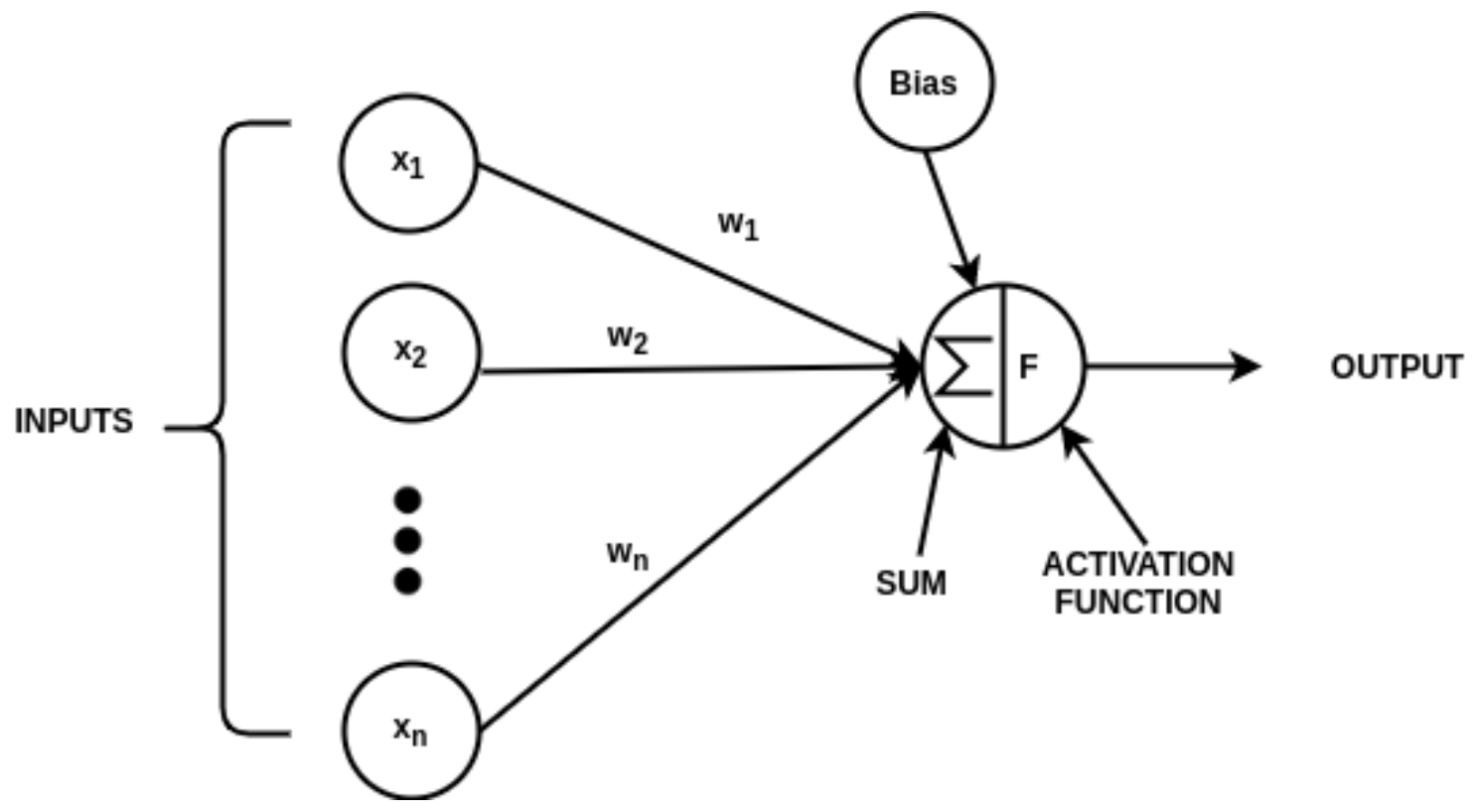

Figure 2.3: Artificial Neuron

There are different activation functions with the purpose of introducing nonlinearity. Some of the most popular activation functions are shown in the table 2.1 . 


\begin{tabular}{|c|c|}
\hline Name & Function \\
\hline Sigmoid $[30]$ & $g(z)=\frac{1}{1+\exp (-z)}$ \\
\hline ReLU $[31]$ & $g(z)=\max (0, z)$ \\
\hline Leaky ReLU $[31]$ & $g(z)=\max (\epsilon * z, z)$ with $\epsilon<<1$ \\
\hline $\operatorname{ELU}[32]$ & $g(z)=\max \left(\alpha\left(e^{z}-1\right), z\right)$ with $\epsilon<<1$ \\
\hline
\end{tabular}

Table 2.1: Activation Functions

\subsubsection{Feedforward Deep Network}

Deep feedforward models or multilayer perceptrons are the typical deep learning models [19]. The goal of these models is to approximate a function $f$ which learns a mapping from input $x$ to the output $y$. The function $y=f(x: \theta)$ is learned by tuning the parameters $\theta$ to find the best function approximation. Figure 2.4 shows a deep feedforward model. The model is used to recognize a person in an image. The example takes raw data pixels as input. Then, it has 3 hidden layers that represent the input from lower to higher features. The first hidden layer may detects edges. The next layer is may detect more complex patterns such as corners. The third layer may learn object parts from features learned from the previous layer. Finally, the last layer is the output layer which recognizes the object by using the high-level features learned in the 3rd hidden layer.

Mathematically, a combination of the weighted sum of the input vector and nonlinear activation function is done to get representation in the first hidden layer. More precisely, this function is defined below:

$$
\begin{gathered}
f^{1}\left(x ; w^{1}, b^{1}\right)=x^{T} w^{1}+b^{1} \\
\left.h^{1}(x)=g\left(f^{1}(x)\right)\right)
\end{gathered}
$$

where $w$ and $b$ are trainable parameters that also are referred as $\theta . \mathrm{g}($.$) is called an$ 


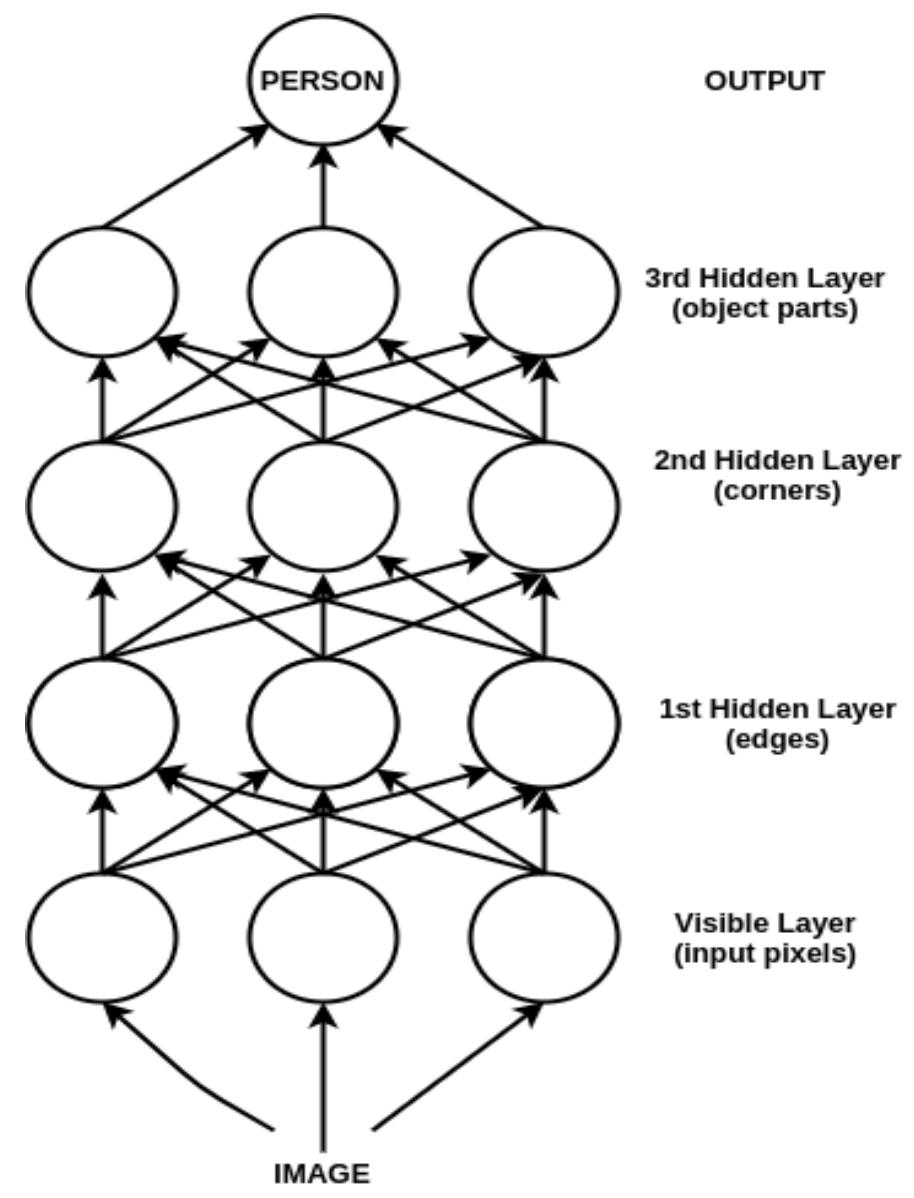

Figure 2.4: Deep Feedforward Networks [19].

activation function that is used to learn non-linear transformation. Rectified linear unit is one of the recommended activation that is defined as $g(z)=\max (0, z)$. 2nd hidden layer takes input from the 1st hidden layer and does a similar transformation. A similar computation is done for the 3rd hidden layer. The equations are as follows:

$$
\begin{gathered}
f^{2}\left(x ; w^{2}, b^{2}\right)=f^{1^{T}} w^{2}+b^{2} \\
\left.h^{2}(x)=g\left(f^{2}(x)\right)\right) \\
f^{3}\left(x ; w^{3}, b^{3}\right)=f^{2 T} w^{3}+b^{3} \\
\left.h^{3}(x)=g\left(f^{3}(x)\right)\right)
\end{gathered}
$$


Finally, the output layer takes the weighted sum of the last hidden layer and computes a loss function or cost function. Here, we compute a sigmoid of the output which converts the output to between 0 to 1 . Figure 2.5 shows the graph of a sigmoid function [30].

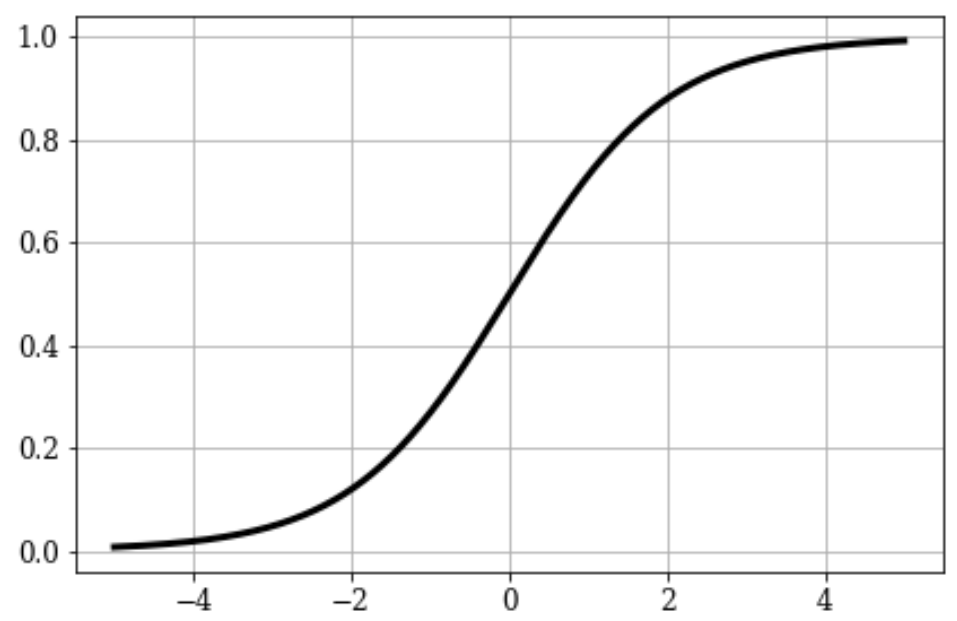

Figure 2.5: Sigmoid Function

$$
\begin{gathered}
o\left(x ; w^{3}, b^{4}\right)=f^{3^{T}} w^{4}+b^{4} \\
\operatorname{sigmoid}(o)=\frac{1}{1+\exp (-o)}
\end{gathered}
$$

If the value of the output of sigmoid is closer to 1 , that means a higher chance of that class to be present in the image. Initially, the output is poor, and to improve the result, the deep feedforward network is trained from the mistakes and the trainable parameters are learned to make a better decision in the future. The process to update the parameters is described in the following sections. 


\subsubsection{Loss Function}

Our deep feedforward network predicts if a person is in the image. It is necessary to define a cost function that computes prediction error rate. If the prediction is false then the error will be more and if it is closer to the right answer the value of error is small. In our example, there are two classes, i.e either there is a person or not. There are many cost functions defined in the literature. Binary Cross Entropy is the most popular cost function and is defined as follows:

$$
\operatorname{Loss}_{b c e}=-\frac{1}{N} \sum_{i=1}^{N} y_{i} \log \left(p\left(y_{i}\right)\right)+\left(1-y_{i}\right) \log \left(1-p\left(y_{i}\right)\right)
$$

where $\mathrm{N}$ is total number of points and the output label $y_{i}$ is 1 if a person is present in the input or 0 otherwise. Thus, depending on the input, one of the two parts of the cost function is relevant and the other is zero. When $y_{i}$ is equal to 1 and our output is also close to 1 then the loss is small. In the cost function, $p\left(y_{i}\right)$ is the probability of the output be a person. We compute this probability by applying sigmoid to the output of the deep feedforward neural network.

\subsubsection{Backpropagation}

To learn a neural network, it is essential to backpropagate [33] the loss to update the weights of the neural network. This is done by minimizing the loss by using an optimizer. To minimize the loss function, the parameters are updated as follows using gradient descent:

$$
\begin{gathered}
w^{l}=w^{l}-\alpha \frac{\partial \text { Loss }}{\partial w^{l}} \\
b^{l}=b^{l}-\alpha \frac{\partial \text { Loss }}{\partial b^{l}}
\end{gathered}
$$


where $\alpha$ is a learning rate and gradients for parameters are computed to update them. Gradient descent is discussed in more detail in the next section.

\subsubsection{Optimizers}

We have established that we want to change weights to decrease the value of the loss. This process is called optimization. To establish the relationship between the loss and parameters we derive the partial derivatives of loss concerning the parameters/weights. From Figure 2.6, one may see that for a positive partial derivative, the weight should be decreased to decrease the value of the loss and for negative partial derivative, the weight should be increased to decrease the value of the loss. In other words, we go down the slope to minimize the loss function.

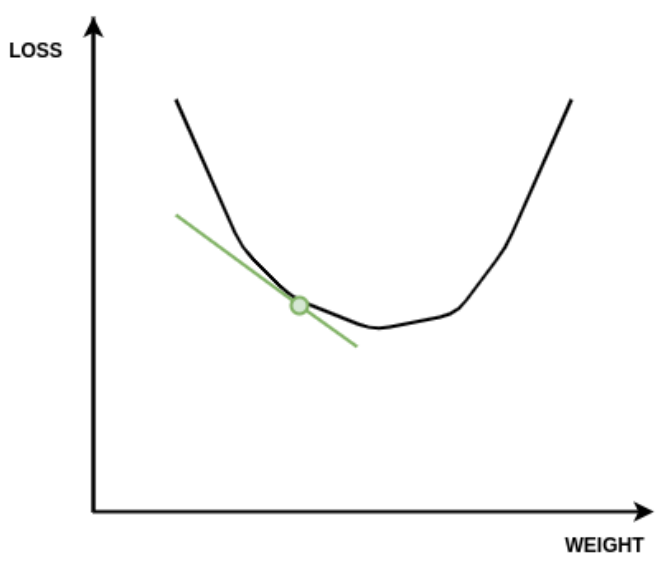

(a)

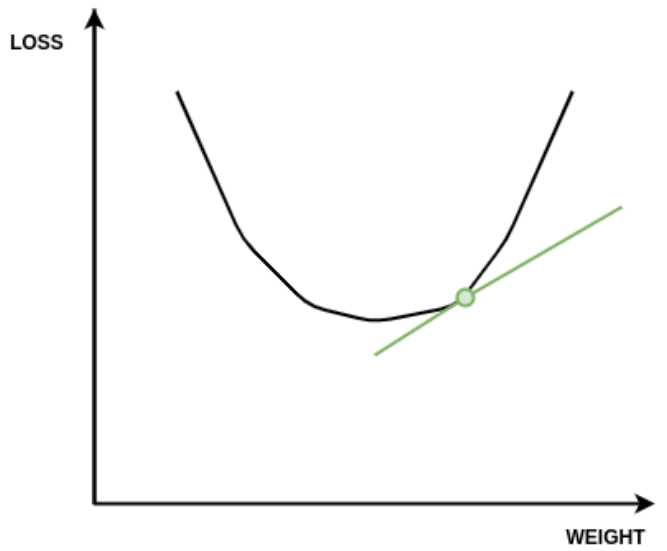

(b)

Figure 2.6: The green point represents the current position for loss and weight. Partial derivative is computed at this point. (a) shows negative partial derivative and (b) shows positive partial derivative

\section{Gradient Descent}

The most basic optimization algorithm that exploits partial derivative is gradient descent. Weights are updated using the following formula in gradient descent algorithm, 


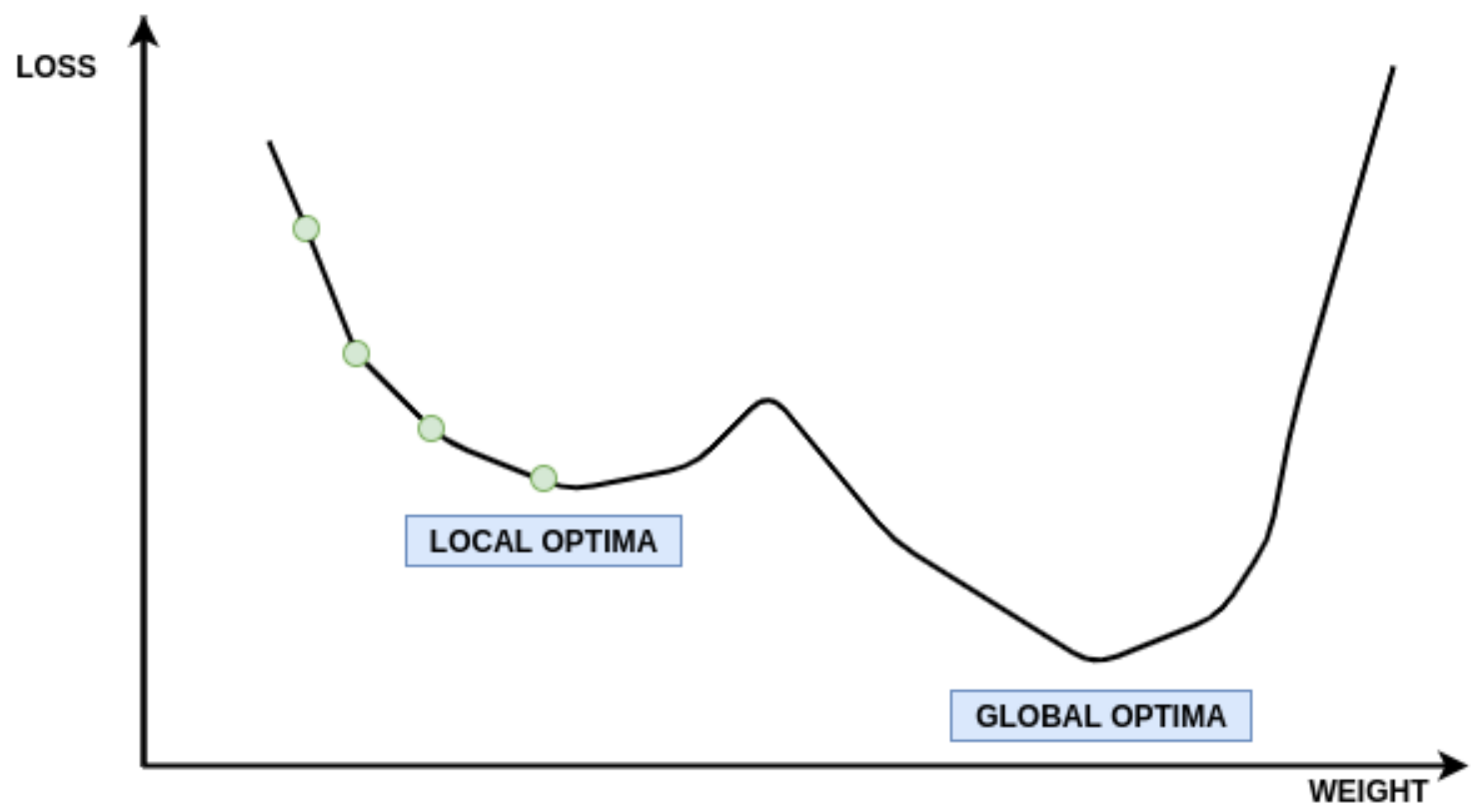

Figure 2.7: The loss can get stuck in local optima with gradient descent

$$
W_{\text {new }}=W_{\text {old }}-\alpha * \frac{\partial \text { Loss }}{\partial W_{\text {old }}}
$$

where, $\alpha$ is the learning rate that controls the speed of updating weights.

Gradient descent optimization is slow because it should be applied to all the data points before doing a single update. A variant of gradient descent, stochastic gradient descent, improves by updating the weights that occur after every training sample. Stochastic gradient descent empirically has been found to converge faster. One of the big problems with gradient descent optimization is that we might get stuck in a local optima as shown in Figure 2.7. This graph is very simple, in reality, there could be multiple local optima.

\section{Momentum}

Momentum [34] is inspired by physics. The updates based on gradient can be considered as a ball rolling down the hill. As the ball roll down the hill, it's momentum 


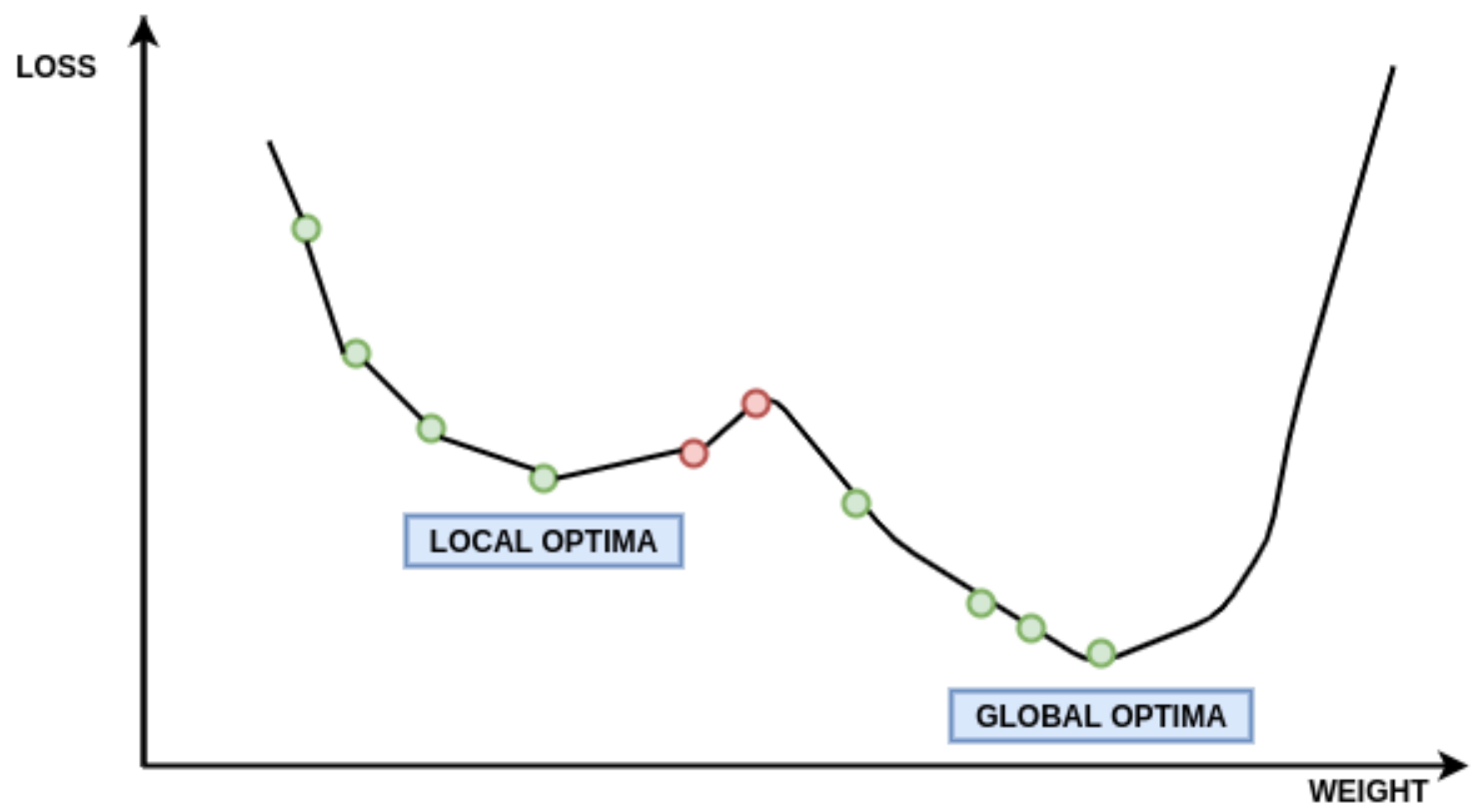

Figure 2.8: The momentum manages to cross the local optima and move toward global optima.

starts to increase. Provided there is enough momentum, when we encounter a local optimum the momentum would provide enough speed to cross the local optima and move towards global optima as demonstrated in figure 2.8. When a local optima is encountered, momentum is decreased due to facing some resistance, as shown by red circles in Figure 2.8, and then it continues going in the original direction.

Mathematically, the weights can be updated using the following equations that include momentum.

$$
\begin{gathered}
v_{\text {new }}=n * v_{\text {old }}-\alpha * \frac{\partial \text { Loss }}{\partial W_{\text {old }}} \\
W_{\text {new }}=v_{\text {new }}+W_{\text {old }}
\end{gathered}
$$

where the momentum $v$ accumulates parts of the previous momentum. $n$ is the coefficient of momentum which decides the amount of momentum that should be 
carried forward.

\section{Adaptive Optimization}

Adaptive optimizers adopt the value of momentum coefficient and learning rate throughout the learning process.

\section{AdaGrad}

Adagrad [35] optimizer changes the learning rate based on the square of gradient value. If the gradient updates are significant then the learning rate will be small and when they are small the learning rate will increase. The following equations are used in Adagrad to update weights.

$$
\text { cache }_{\text {new }}=\text { cache }_{\text {old }}+\left(\frac{\partial(\text { Loss })}{\partial\left(W_{\text {old }}\right)}\right)^{2}
$$

The cache stores the accumulated squares of the gradients from the beginning of the training.

$$
W_{\text {new }}=W_{\text {old }}+\frac{\alpha}{\sqrt{\text { cache } e_{\text {new }}}+\epsilon} * \frac{\partial(\text { Loss })}{\partial\left(W_{\text {old }}\right)}
$$

where $\epsilon$ is added to avoid division by zero. The value of the cache affects the learning rate. The drawback of this formulation is that cache will only increase as the training proceeds because the square of input will always be positive. Hence, the learning rate will keep on decreasing and move closer to zero and training becomes very slow. RMSProp optimizer slows the decrease in learning rate per weight update.

\section{RMSProp}

RMSProp [36] improves the cache update equation by introducing a new parameter called delay rate or gamma. 


$$
\text { cache }_{\text {new }}=\gamma * \text { cache }_{\text {old }}+(1-\gamma) *\left(\frac{\partial(\text { Loss })}{\partial\left(W_{\text {old }}\right)}\right)^{2}
$$

where the value of $\gamma$ is generally 0.9 which helps to slow down the decay in the learning rate. Consequently, the training continues for a longer period.

\section{ADAM}

ADAM [37] is one of the most popular optimizers to date that combines momentum with RMSprop. The update formulas with ADAM are:

$$
\begin{gathered}
m_{\text {new }}=\beta_{1} * m_{\text {old }}-\left(1-\beta_{1}\right) * \frac{\partial(\text { Loss })}{\partial\left(W_{\text {old }}\right)} \\
\text { cache }_{\text {new }}=\beta_{2} * \text { cache }_{\text {old }}+\left(1-\beta_{2}\right) *\left(\frac{\partial(\text { Loss })}{\partial\left(W_{\text {old }}\right)}\right)^{2} \\
W_{\text {new }}=W_{\text {old }}-\frac{\alpha}{\sqrt{\text { cache } e_{\text {new }}}+\epsilon} * m_{\text {new }}
\end{gathered}
$$

with these update rules, ADAM adapts momentum and learning rate in every update. The recommended settings for $\beta_{1}$ and $\beta_{2}$ is 0.9 and 0.99 respectively.

\subsection{Convolutional Neural Networks}

Convolutional Neural Networks (CNNs) [38] are called convolutional because they employ the convolution operation instead of matrix multiplication used in regular neural networks. Basic layers often used in building a CNN are shown in Figure 2.9. The input image is passed through convolutional and pooling layers to learn features from the input. These features are then passed through a regular fully connected neural network.

Convolution and pooling layers are discussed in the next section. 


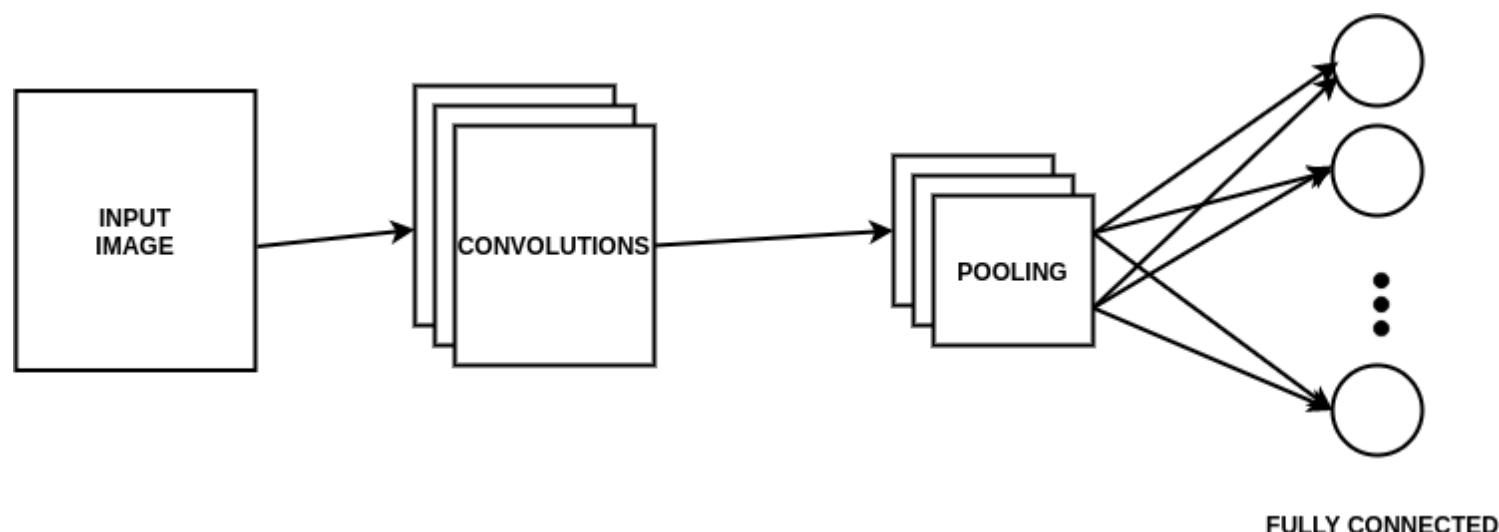

Figure 2.9: General layers in a Convolutional Neural Network.

\subsubsection{Convolution Layer}

A convolutional layer is built from a set of learnable filters or kernels. Each kernel is applied to input. For example, Figure 2.10 shows how a kernel of size 3x3 is applied to an input image of size $5 \times 5$. The output is obtained by applying the convolution operation between the image and the filter. The value in the kernel is multiplied with the values of an input image and the sum of those values gives an output. The same kernel is applied to other points in the input image by sliding the kernel on top of the input image. Stride is the number of pixels the filter moves to compute the next values. The value of stride affects the dimension of the output. In our example, the value of stride is set to 1 .

\begin{tabular}{|c|c|c|c|c|c|c|c|}
\hline 1 & 0 & $1-$ & -0 & -0 & -1 \\
\hline 1 & 0 & 0 & 1 & 1 \\
\hline 1 & 1 & 0 & 0 & 1 \\
\hline 0 & 1 & 0 & -1 & -0 \\
\hline 0 & 0 & 1 & 1 & 0 \\
\hline
\end{tabular}

KERNEL

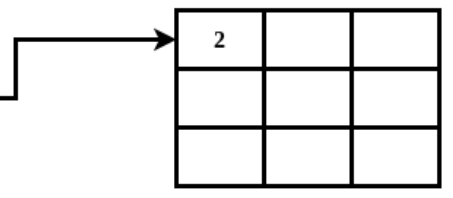

OUTPUT

INPUT IMAGE

Figure 2.10: Convolution operation 


\section{Output Dimension}

A convolution layer consists of multiple kernels that learn different features like edges, corners, etc and each kernel outputs a feature map. Thus, the depth of the output is equal to the number of kernels in the convolution layer. The width and height of the output are dependent on the stride. The equation to compute the output width and height is,

$$
\begin{gathered}
\text { output_width }=\left\lfloor\frac{\text { input_width }- \text { kernel_width }}{\text { stride }}\right\rfloor+1 \\
\text { output_height }=\left\lfloor\frac{\text { input_height }- \text { kernel_height }}{\text { stride }}\right\rfloor+1
\end{gathered}
$$

Using the above equations, we can compute the output dimension of 2.10 .

output_width $=\frac{5-3}{1}+1=3$

output_height $=\frac{5-3}{1}+1=3$

Thus, the output dimension is $3 \times 3 \times 1$. Depth is 1 because in our example we use 1 kernel.

\section{Padding}

In 2.10, if the dimension of input were $6 \times 6$ and stride 2 , then, the dimensions for the last convolution would not match. In this scenario, the last convolution is dropped. The output dimension would be $2 \mathrm{x} 2$ with some loss of information. To overcome the loss of information, the input is padded with zeroes. Figure 2.11 shows padding with $P_{\text {start }}$ as the padding at the beginning of input shown in red color and $P_{\text {end }}$ as the padding at the end of an input shown in blue color.

Padding has mainly two benefits: it allows using all the information of the input and this helps to control the dimension of the output. The padding that controls the output dimension is called the SAME padding. In this mode of padding, the 


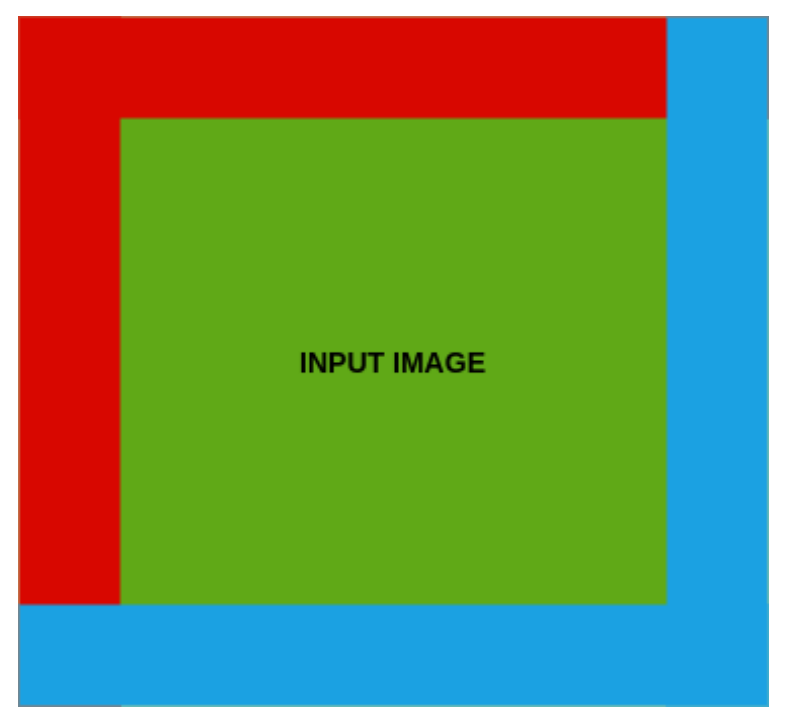

Figure 2.11: Padded input at beginning with length equal to $P_{\text {start }}$ shown in red and at the end with length equal to $P_{\text {end }}$ shown in blue.

dimension of the output is the ceiling of the ratio of the input size to the stride. Mathematically, the amount of padding can be calculated as follows:

$$
P_{\text {start }}=\left\lfloor\frac{S\left\lceil\frac{I}{S}\right\rceil-I+F-S}{2}\right\rfloor
$$

where, $\mathrm{S}$ is stride, I is the input length and $\mathrm{F}$ is kernel/filter length. In the case of a $6 \times 6$ input and a kernel size of $3 \times 3$, when the stride is 2 , the resulting output dimension will be $3 \times 3$. This is because the input width and height is modified after padding. The updated equation to compute the output dimension with padding is:

$$
\text { output_length }=\left\lfloor\frac{I-F+P_{\text {start }}+P_{\text {end }}}{S}+1\right\rfloor
$$

Many times $P_{\text {start }}=P_{\text {end }}$, in that case $P_{\text {start }}+P_{\text {end }}$ can be replaced with $2 \mathrm{P}$.

\subsubsection{Pooling Layer}

The pooling layer applies a function to the input to reduce the spacial size of the representation which helps to control overfitting by reducing the number of parameters 
in the network. The pooling layer is applied to every slice of the image separately to downsample the input. There are mainly two types of pooling layer: max and average. Figure 2.12 demonstrates max-pooling which has a size of 2x2 with a stride of 2 . For every $2 \times 2$ window, the max element is selected in the max-pooling layer. Similarly, in the case of average pooling, the average of the samples is computed.

\begin{tabular}{|l|l|l|l|}
\hline 4 & 6 & 2 & 4 \\
\hline 2 & 7 & 8 & 2 \\
\hline 4 & 2 & 2 & 6 \\
\hline 4 & 2 & 1 & 5 \\
\hline
\end{tabular}

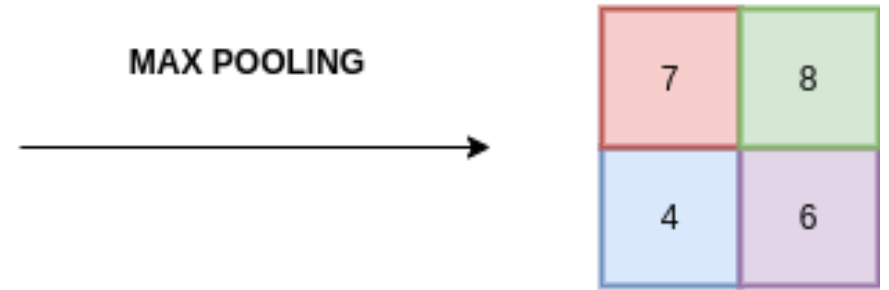

Figure 2.12: Max Pooling

\subsubsection{Properties}

Unlike regular neural networks, the convolutional neural network has properties like sparse interactions and parameter sharing. We will discuss these properties in this section.

\section{Sparse Interactions}

In regular neural networks, every input is connected with every output. However, in CNN the input is connected with a very small amount of output node. The sparsity is decided by the kernel size. The output node, on the other hand, is also connected with a small amount of input node which is considered the receptive field for the output node. 


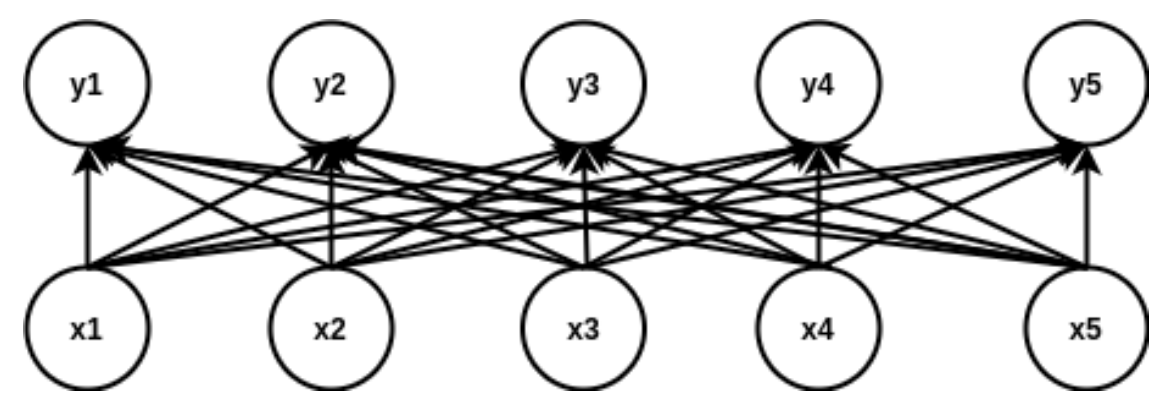

(a) Regular Neural Network

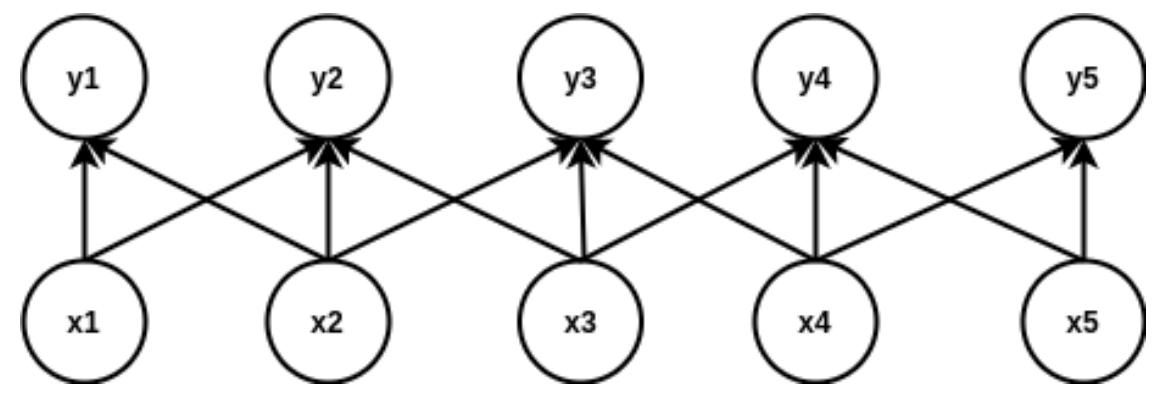

(b) Convolutional Neural Network

Figure 2.13: (a) is a regular neural network. (b) is a convolutional neural network with kernel width of 3 [19].

An example for illustrating sparse interactions is shown in Figure 2.13. The example demonstrates that the input node $\mathrm{x} 3$ can only effect output node y2,y3 and y4 and output node $\mathrm{y} 3$ has input $\mathrm{x} 2, \mathrm{x} 3$ and $\mathrm{x} 4$ in it's receptive field.

\section{Parameter sharing}

In a regular neural network, no parameter is shared and every weight is used once. However, in convolutional neural network same kernel is applied to the input by sliding it with steps specified by the value of stride. This leads to a much smaller number of trainable parameters and needs much less memory space to store them. Thus, CNN is more efficient in terms of memory requirements. 


\subsubsection{Popular Architectures}

LeNet [39] is one of the first CNN architectures designed for handwritten digit recognition. AlexNet [40] introduced in 2012, is a similar architecture but is deeper with more filters per layer. This architecture also started using the ReLU activation function instead of the Sigmond function. Furthermore, AlexNet is designed in a way that the model can be split and placed into two GPUs to speed up the training process. This network has about 60 million parameters. Google introduced Inception network with much less number of parameters (about 4 million) and outperformed AlexNet in ILSVRC 2014. VGGNet [41] is similar to AlexNet but has more filters. This leads to 138 million parameters which require a lot of training data and time to train. Till now it was assumed that deeper models perform better. However, one issue with very deep structures is the information about the loss may not travel back efficiently to the initial layers. Resnet [42] introduced skip connections to overcome this issue. 


\section{Chapter 3}

\section{Related Work}

The proposed method may be seen as a deep extension of the k-NN classifier. In this chapter, we first review the previous attempts is combining k-NN and neural networks. The proposed method is based on learning a latent space where k-NN can be applied. Therefore, it is related to the broader family of metric learning methods. In Section 3.2, we will discuss related work in deep metric learning.

\subsection{Deep Extensions of Nearest Neighbors}

There have been several previous attempts at the intersection of k-NN and neural networks.

\section{Deep k-nearest neighbors}

Papernot and McDaniel introduced Deep k-nearest neighbors (DkNN) [43] which applies an ensemble of k-NN classifiers to the activations at intermediate layers of a neural network to measure uncertainty and improve the robustness against adversarial attacks. DkNN enforces conformity of the predictions made by a DNN which provides protections against adversarial test inputs. DkNN performs a nearest neighbor search at each layer of DNN to find the nearest points in the training point. The labels 
of these points are analyzed to make sure that intermediate representation remains conformal with the final prediction. DkNN is interpretable because the output is provided by using the nearest neighbors. The method has high confidence when there is homogeneity between the nearest neighbor labels. When the intermediate representation is not conformal it is generally due to adversarial examples. Thus, this method is robust to adversarial examples. For an input $x$ with the label $j$ nonconformity measure is computed using,

$$
\alpha(x, j)=\sum_{\lambda \in 1 . . l}\left|i \in \omega_{\lambda}: i \neq j\right|
$$

where $l$ is the number of intermediate layers/representation and $\omega_{\lambda}$ is the multi-set of labels for the nearest training points.

DkNN does not offer a loss function. It can be seen as a post-hoc mechanism for improving the robustness of a neural network classifier against adversarial attacks. The neural network itself needs to be trained separately using common loss functions such as cross-entropy.

\section{Neural Nearest Neighbors Networks}

One of the main challenges in optimizing for $\mathrm{k}-\mathrm{NN}$ is imposed by the nondifferentiability of the k-NN. Plötz and Roth [44] tried to address this by defining the nearest neighbor as a weighted sum of the training samples where the weights are proportional to the negative distances to the query. The authors introduced the neural nearest neighbor block ( $\mathrm{N}^{3}$ block) for non-local processing which can be used for exploiting self-similarity, for example, in image denoising.

As shown in Figure 3.1, $N^{3}$ block takes the output of a neural network layer as input. Embedding network computes feature embeddings using a multilayer perceptron. The pairwise distance matrix D is computed from the output of the embedding 


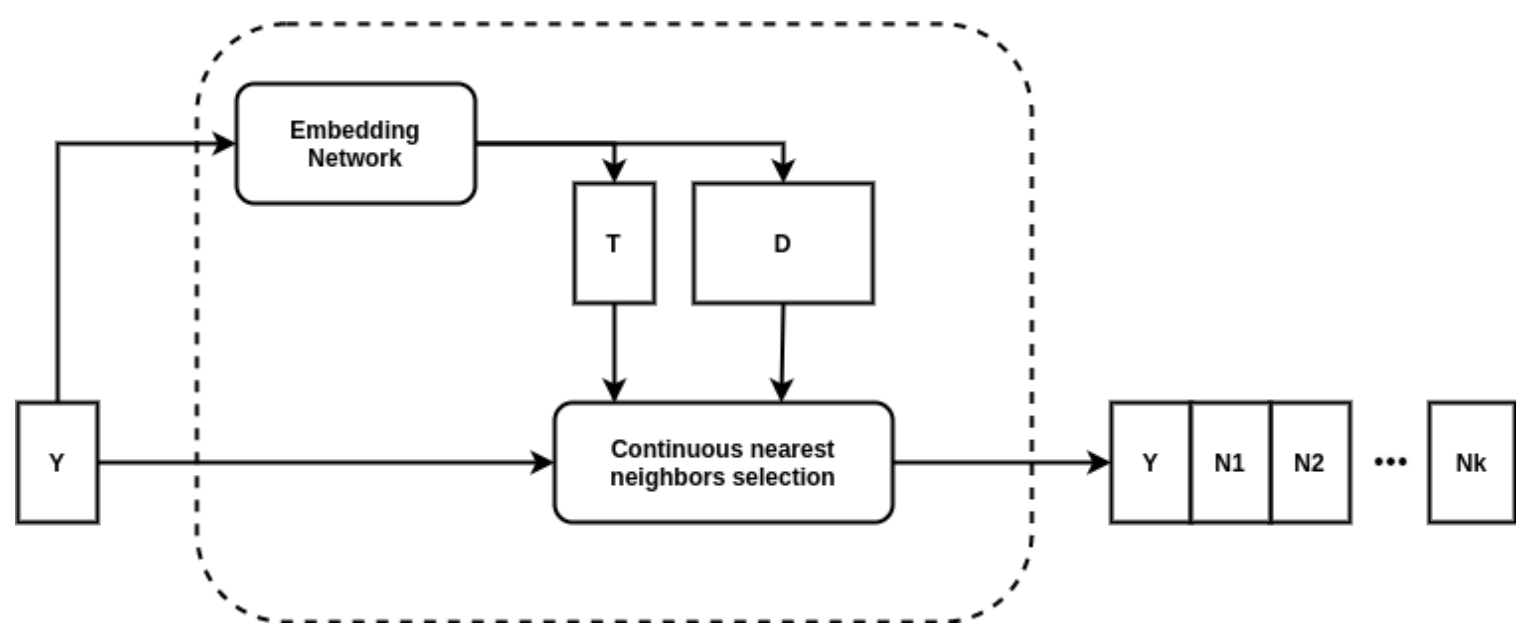

Figure 3.1: $N^{3}$ block

network. The distance function used in their work is Euclidean distance. Another network $\mathrm{T}$ computes the temperature for each item. Embedding network and $\mathrm{T}$ are trained separately without sharing weights. Distance matrix D and temperature tensor $\mathrm{T}$ are used to compute $\mathrm{k}$ continuous nearest neighbors feature volumes $N_{1}, N_{2}, \ldots, N_{k}$ which is done using continuous deterministic relaxation for discrete variables to allow backpropagation through the neighborhood selection process.

However, $\mathrm{N}^{3}$ does not offer a loss function. Indeed it uses mean square error loss for training neural networks augmented with ( $\mathrm{N}^{3}$ block) blocks. Due to the lack of a loss function, another loss function is required to optimize the network architecture.

\section{Soft Nearest Neighbors}

Salakhutdinov and Hinton introduced soft nearest neighbor (SNN) for training neural networks [45]. Recently Frosst et.al [46] demonstrated several use cases of the SNN loss. MsDNN is related to the SNN loss in that both compute a notion of the probability of being the nearest sample. The SNN loss is defined as, 


$$
\mathcal{L}_{s n}(x, y, T)=-\frac{1}{b} \sum_{i \in 1 \ldots b} \log \left(\frac{\sum_{\substack{j \in 1 . . . b \\ j \neq i \\ y_{i}=y_{j}}} e^{-\frac{\left\|x_{i}-x j\right\|^{2}}{T}}}{\sum_{\substack{k \in 1 . . b \\ k \neq i}} e^{-\frac{\left\|x_{i}-x k\right\|^{2}}{T}}}\right)
$$

for temperature $\mathrm{T}$ and batch $b$ on samples $(x, y)$ where $x$ represents the raw input vector, and $y$ represents the labels. When the temperature is low, the loss puts more weight on small distances. On the other end, when the temperature is high widely separated points can influence the soft nearest neighbor loss.

However, similar to the $\mathrm{N}^{3}$ method [44], the proposed method aggregates the features of the neighbors while SNN aggregates the labels of the neighbors. This allows us to explicitly define sample margin and relate it to the expected LOO 1NN classification error; -i.e. a sample is misclassified if its margin is negative. The proposed loss maximizes the margin of a sample only if the sample is misclassified. In contrast, SNN does not have such a selective update mechanism.

\subsection{Deep Metric Learning}

The purpose of deep metric learning is to minimize the distance between samples from the same classes and maximize the distance between samples from different classes. Deep metric learning utilizes deep architectures to obtain embeddings in latent space which are procured through non-linear transformation. In this section, loss functions commonly used for deep metric learning are discussed.

\subsubsection{Contrastive Loss}

Contrastive loss $[21,47]$ is applied in the literature to pairs of samples to minimize or maximize the distance between them according to their labels. The loss function is 


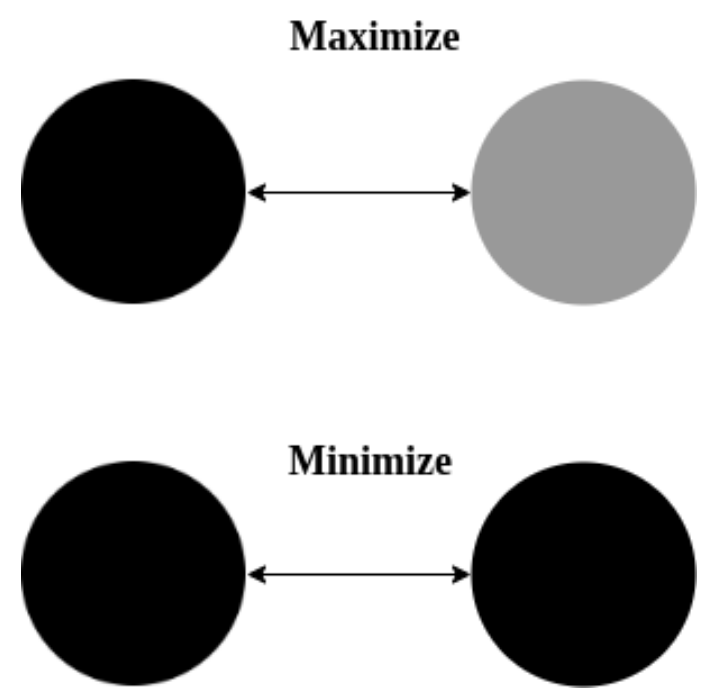

(a) Contrastive Loss

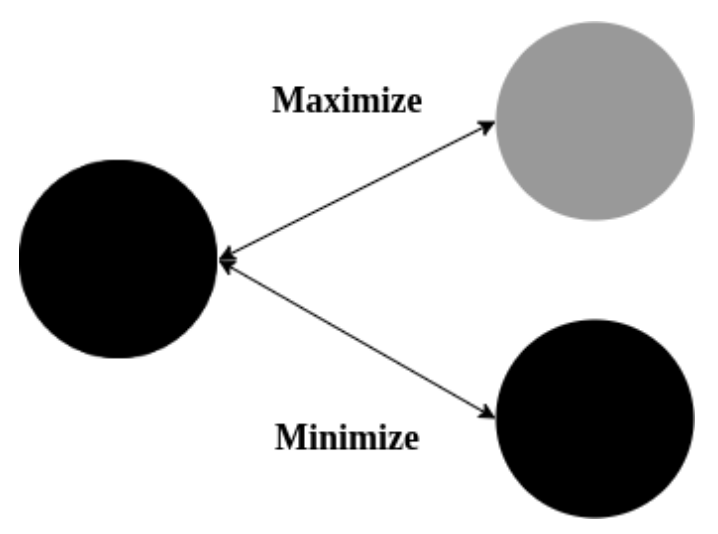

(b) Triplet Loss

Figure 3.2: Contrastive loss looks at absolute distance and Triplet Loss looks at relative distance

defined as,

$$
\mathcal{L}_{\text {Contrastive }}=\frac{1}{m} \sum_{(i, j)}^{m / 2} y_{i, j} D_{i, j}^{2}+\left(1-y_{i, j}\right)\left[\alpha-D_{i, j}\right]_{+}^{2}
$$

where, $m$ is the number of samples. Distance between a pair of samples is calculated in the embedding space using $D_{i, j}^{2}=\left\|f\left(x_{i}\right)-f\left(x_{j}\right)\right\|_{2}$ where $\mathrm{f}($.$) is the output of$ the backbone network [47] and the label $y_{i, j} \in\{0,1\}$ is used to inform if the samples inside a pair are from same class or different classes. The $[.]_{+}=\max (0,$.$) operator is$ the hinge function.

In other words, the contrastive loss minimize the distance between samples when the label is 1 (same class) and maximize the distance between samples for being smaller than the margin parameter $\alpha$ when label is 0 (different class).

The drawback of contrastive loss is that it is applied to absolute distance and not relative distance. 


\subsubsection{Triplet Loss}

Triplet loss $[3,22]$ unlike contrastive loss, has three objects which are anchor, positive and negative samples as shown in Figure 3.2. It aims to increase the distance between the anchor and negative samples and decrease the distance between the anchor and the positive sample. Furthermore, it also is optimized to make sure that the distance between the anchor and negative is more than the anchor and positive. The triplet loss minimizes the following loss function,

$$
\mathcal{L}_{\text {Triplet }}=\frac{1}{T} \sum_{(i, j, k \in T)}\left[D_{i, j}^{2}+\alpha-D_{i, k}^{2}\right]_{+}
$$

where, $\mathrm{T}$ is the number of triplets and distance between two samples is computed using the equation, $D_{i, j}^{2}=\left\|f\left(x_{i}\right)-f\left(x_{j}\right)\right\|_{2}$ where $\mathrm{f}($.$) is the output of network. The$ $[\cdot]_{+}=\max (0,$.$) operator is the hinge function and \alpha$ denotes a margin.

\section{Sampling Issue}

The number of possible triplets for Triplet loss is $O\left(n^{3}\right)$. This is a major problem in this setup, as it is not possible to consider all the triplets due to poor speed and the fact that most of these triplets don't help the model to learn discriminative features. Thus, many methods are proposed in the literature to solve the issue of sampling triplets.

Facenet [3] mined triplets following an online strategy. It constructs triplets by finding a semi-hard negative example for a positive pair in a mini-batch. For a positive pair, the semi-hard negative example is defined as the sample whose distance from the anchor is more than the positive sample but is within the margin as shown in Figure 3.3.

In order to get many triplets which are not easy triplets, this approach needs very large mini-batches. Thus, it is hard to train it on GPU due to memory constraints. 


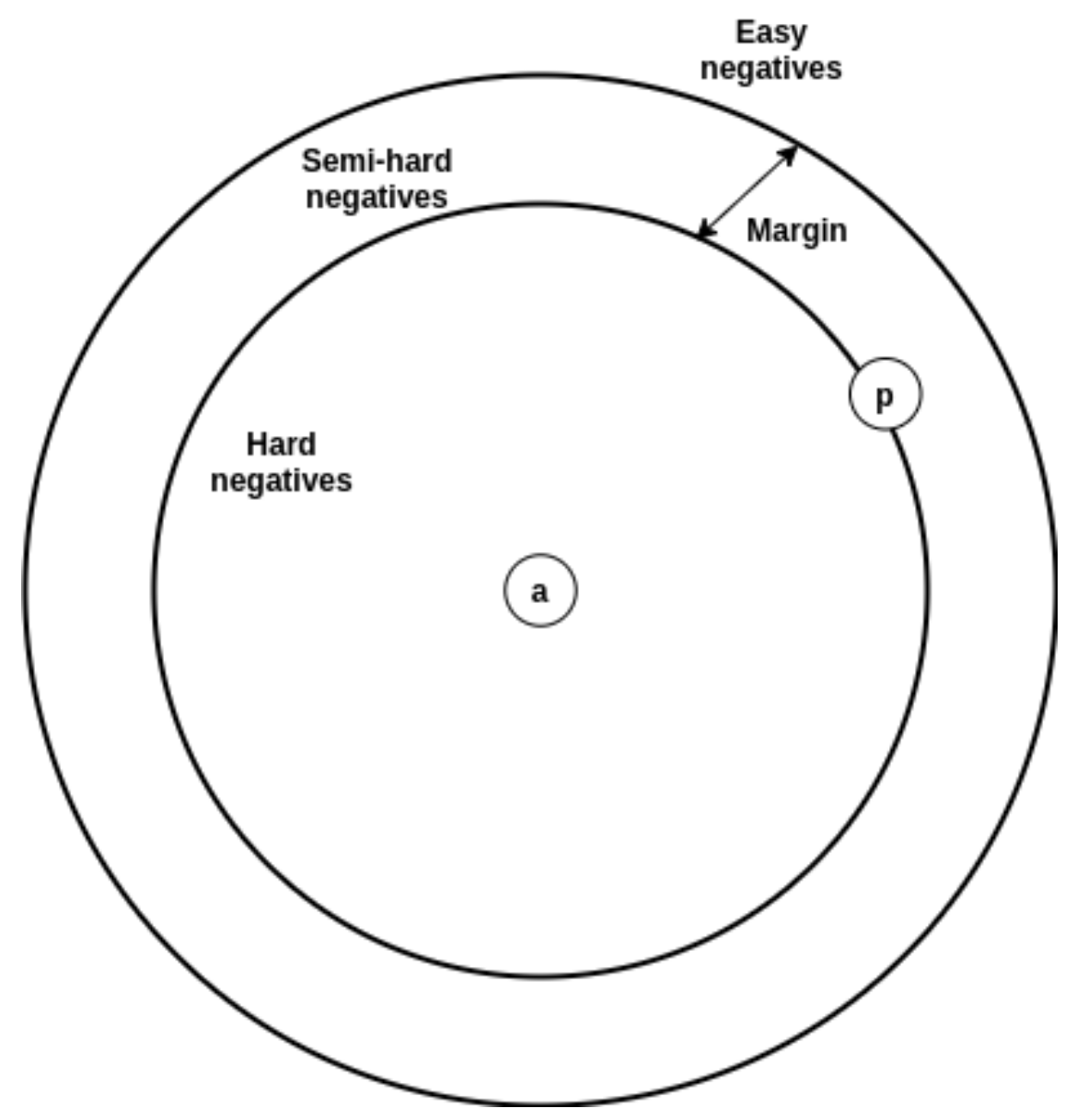

Figure 3.3: Semi-Hard Triplet Mining

The paper [26] proposed a differentiable smooth loss by incorporating all negative examples called lifted structured loss. The aim is to pull a positive pair as close as possible and push all negative samples corresponding to one of the positive pair point farther than the margin. Concretely, the lifted structured loss function can be written as follows,

$$
\begin{gathered}
\mathcal{L}_{\mathrm{ij}}=d_{i j}+\log \left(\sum_{(i, k) \in N} \exp \left(\alpha-d_{i k}\right)+\sum_{(j, l) \in N} \exp \left(\alpha-d_{j l}\right)\right) \\
\mathcal{L}_{\text {lifted }}=\frac{1}{2|P|} \sum_{(i, j) \in \mathcal{P}} \max \left(0, \mathcal{L}_{i j}\right)^{2}
\end{gathered}
$$


where $\mathrm{N}$ and $\mathrm{P}$ are sets of negative and positive pairs respectively.

\subsubsection{Other methods inspired by Triplet loss}

Triplet loss pulls one positive towards the anchor and pushes one negative away from the anchor, even in the case of a multi-class problem. In multi-class problem with $\mathrm{N}$ classes there are N-1 classes that are different from anchor. In N pair loss [23], one positive sample and N - 1 negative samples are considered where $\mathrm{N}$ is the number of classes for a given query or anchor. One sample is randomly selected per negative class which is pushed away from the anchor. The loss function is define as,

$$
\mathcal{L}_{\mathrm{N}-\text { pair-mc }}=\frac{1}{N} \sum_{(i=1)}^{N}\left[\log \left(1+\sum_{j !=i} \exp \left(f_{i}^{T} f_{j}^{+}-f_{i}^{T} f_{i}^{+}\right)\right)\right]
$$

where $f_{i}^{T}$ is the query, $f_{i}^{+}$is positive sample and $f_{j}^{+}$is negative sample. This loss is dependent on the number of classes. The performance decreases with the number of classes.

Angular loss [24] encodes a third-order relation inside the triplet triangle which is an angle at the negative edge, instead of focusing on distance between pairs or triplets. The loss aims to push the negative feature vector away from the center of the positive cluster and brings the positive pair closer together.

The above methods are tackling the consequences of a triplet model that has $\mathrm{O}\left((\mathrm{p}-1)^{*} \mathrm{q}\right)$ triplet combinations for a query image/anchor where $\mathrm{p}$ is the number of samples in the same class and $q$ is the number of samples in different classes. Instead, we can focus on all the positive and negative sample's effect on a query image. Our method weights every sample based on how similar it is to the query image. Higher weight is given to the samples that are the nearest and similarity is defined in terms of euclidean distance.

Another drawback of these methods is that they focus on keeping the samples 
from the same class (not samples that look similar) nearby even at the expense of distorting the space to the point where the intrinsic similarity is lost and subclasses in the class are merged. To keep the intrinsic similarity, our method puts more weight on nearby positive samples and keeps them close. 


\section{Chapter 4}

\section{Proposed Method}

\subsection{Introdution}

One of the main problems with classifiers in the field of deep learning is the lack of interpretability. $\mathrm{k}-\mathrm{NN}$ is an interpretable classifier because the prediction may be interpreted from the labels of the nearest neighbors of a query sample. Performance of $\mathrm{k}-\mathrm{NN}$ classifier is dependent on the representation of data. To learn a good representation of data for the $\mathrm{k}-\mathrm{NN}$ classifier, distance metric learning is used where examples from the same class are nearby and examples from the different classes are further apart. There are many methods in literature to learn the representation of data or embedding space using metric learning. These methods learn a space which is optimized to have separate classes. The mainstream approaches assume each class has a single mode. It is essential to learn a space that separates the data based on classes and preserves the similarity between inputs by allowing disjoint subclasses. By learning such an embedding space, the classifier is not only interpretable but also can be used to discover subclasses or clusters within the classes originally fed to the classifier.

In this chapter, we present our proposed method called Multi-scale Deep Nearest Neighbor (MsDNN). 
For a query sample or anchor, the nearest hit is the nearest neighbor from the same class and the nearest miss is the nearest neighbor from a different class. The combination of anchor, nearest miss (NM), and nearest hit (NH) is called a triplet.

For a triplet, MsDNN loss is designed to bring the nearest hit more closer to the anchor than the nearest miss. When we make a prediction using a 1-NN classifier, the predicted class of the test data is the same as that of the nearest sample. A classification error occurs when the nearest neighbor does not have the same label as the anchor. That is there is a closer sample with a different label. When a classification error occurs, it reports a flaw in the embedding space. This flaw can be corrected by moving the nearest sample of the same class closer to the test data than the nearest sample from a different class. MsDNN loss optimizes the embedding space to do the same by moving the NH closer and NM further away from the anchor. Therefore, MsDNN minimizes the classification error of the 1-NN classifier. Furthermore, during training, every sample can be treated as an anchor in a leave-one-out cross-validation fashion. Thus, MsDNN loss is designed to learn a non-linear transformation using a neural network to minimize the leave-one-out classification error of a 1-NN classifier.

A nearest neighbor classifier that looks at only the nearest sample is very local and the decision boundary is not usually smooth. For large values of $\mathrm{K}$, the model becomes too simple and underfits. It is important to find the value to $\mathrm{k}$ to prevent overfitting or underfitting.

For test data in the k-NN classifier, the $\mathrm{k}$ nearest neighbors are at different distances but contribute equally in prediction through majority voting. This is not desired specially when the neighbors vary widely in distance from the anchor. Samples closer to the nearest neighbor should have a larger effect. To solve this issue, k-NN is modified to a weighted nearest neighbor where each neighbor has a weight corresponding to the influence it has in making the prediction. The weights are inversely proportional to the distance from the query sample. Many kernels are suggested to 
define the relation between weights and distance. One popular choice is a Gaussian kernel. Unlike $\mathrm{k}-\mathrm{NN}$ that works with discrete values of $\mathrm{k}$, in the weighted nearest neighbor the scale parameter of the Gaussian kernel can vary continuously. In the weighted nearest neighbor, the scale parameter is tuned to adjust the weight decay. Weight decays with the distance and becomes zero for sufficiently far samples. In $\mathrm{k}-\mathrm{NN}$, the first $\mathrm{k}$ neighbors have the same weight and every other sample has zero weight. Thus, in k-NN, there is a sudden drop in the value of weight assigned whereas the drop of weights in the weighted nearest neighbor is continuous.

In MsDNN, we define $\mathrm{NM}$ and $\mathrm{NH}$ as the weighted average of all the examples from different classes and the weighted average of all the examples from the same class respectively. The weights are inversely proportional to the distances from the anchor and decay with a scale parameter called sigma. In the following we will present our proposed method.

\section{2 $\mathrm{MsDNN}$}

Consider an embedding space defined by a mapping function $\mathbf{f}$ parameterized with $\boldsymbol{\theta}$. Following [48], the margin of an input sample $\mathbf{x}^{(i)}$ in the embedding space can be defined as follows.

$$
\mathbf{r}^{(n)}(\theta)=d\left(\mathbf{f}_{\theta}\left(\mathbf{x}^{(n)}\right), \mathbf{f}_{\theta}\left(\mathrm{NM}\left(\mathbf{x}^{(n)}\right)\right)\right)-d\left(\mathbf{f}_{\theta}\left(\mathbf{x}^{(n)}\right), \mathbf{f}_{\theta}\left(\mathrm{NH}\left(\mathbf{x}^{(n)}\right)\right)\right)
$$

where $\operatorname{NM}\left(\mathbf{x}^{(i)}\right)$ is the nearest neighbor of $\mathbf{x}^{(i)}$ with a different class label (nearest miss) and $\mathrm{NH}\left(\mathbf{x}^{(i)}\right)$ is the nearest neighbor of $\mathbf{x}^{(i)}$ with the same class label as $\mathbf{x}^{(i)}$ (nearest hit). $d(\cdot)$ is a distance function. Here, we use Euclidean distance. Intuitively, a positive margin for a sample means that the sample will be correctly classified using a 1-NN classifier when we leave that sample out. Ideally, the parameters $\theta$ should be learned such that $\mathbf{r}^{(n)}(\theta)$ be positive for all samples, i.e. zero LOO 1-NN 
classification error rate. The margin defined in (4.1) is quite discontinuous and hence hard to optimize. To alleviate this, following [44], we define the nearest sample as the expectation over all possible candidates for being the nearest sample and define the expected margin for the $\mathrm{n}$-th sample as follows.

$$
\begin{aligned}
\overline{\mathbf{r}}^{(n)}(\theta)=\left\|\mathbf{f}_{\theta}\left(\mathbf{x}^{(n)}\right)-\mathbb{E}_{i \sim \mathcal{M}_{n}} \mathbf{f}_{\theta}\left(\mathbf{x}^{(i)}\right)\right\|_{2}^{2}-\left\|\mathbf{f}_{\theta}\left(\mathbf{x}^{(n)}\right)-\mathbb{E}_{i \sim \mathcal{H}_{n}} \mathbf{f}_{\theta}\left(\mathbf{x}^{(i)}\right)\right\|_{2}^{2} \\
=\left\|\mathbf{f}_{\theta}\left(\mathbf{x}^{(n)}\right)-\sum_{i \in \mathcal{M}_{n}} P\left(\mathbf{x}^{(i)}=\operatorname{NM}\left(\mathbf{x}^{(n)}\right) \mid \theta\right) \mathbf{f}_{\theta}\left(\mathbf{x}^{(i)}\right)\right\|_{2}^{2} \\
\quad-\left\|\mathbf{f}_{\theta}\left(\mathbf{x}^{(n)}\right)-\sum_{i \in \mathcal{H}_{n}} P\left(\mathbf{x}^{(i)}=\operatorname{NH}\left(\mathbf{x}^{(n)}\right) \mid \theta\right) \mathbf{f}_{\theta}\left(\mathbf{x}^{(i)}\right)\right\|_{2}^{2}
\end{aligned}
$$

$\mathcal{M}_{n}$ and $\mathcal{H}_{n}$ denote the set of all possible candidates for $\operatorname{NM}\left(\mathbf{x}^{(n)}\right)$ and $\mathrm{NH}\left(\mathbf{x}^{(n)}\right)$ respectively and are defined as $\mathcal{M}_{n}=\left\{j \in\{1, \ldots, M\} \mid y^{(j)} \neq y^{(n)}\right\}$ and $\mathcal{H}_{n}=$ $\left\{j \in\{1, \ldots, M\} \mid y^{(j)}=y^{(n)}, j \neq n\right\}$.

$\mathbb{E}_{j \sim \mathcal{M}_{n}}$ denotes the expectation computed with respect to $\mathcal{M}_{n}$.

$P\left(\mathbf{x}^{(i)}=\operatorname{NM}\left(\mathbf{x}^{(n)}\right) \mid \theta\right)$ and $P\left(\mathbf{x}^{(i)}=\mathrm{NH}\left(\mathbf{x}^{(n)}\right) \mid \theta\right)$ are the probabilities of a sample $\mathbf{x}^{(i)}$ being the nearest miss or hit of $\mathbf{x}^{(n)}$, respectively. They can be estimated via the standard kernel density estimation.

$$
\begin{gathered}
P\left(\mathbf{x}^{(i)}=\operatorname{NM}\left(\mathbf{x}^{(n)}\right) \mid \theta\right)=\frac{k\left(d\left(\mathbf{f}_{\theta}\left(\mathbf{x}^{(n)}\right), \mathbf{f}_{\theta}\left(\mathbf{x}^{(i)}\right)\right)\right)}{\sum_{j \in \mathcal{M}_{n}} k\left(d\left(\mathbf{f}_{\theta}\left(\mathbf{x}^{(n)}\right), \mathbf{f}_{\theta}\left(\mathbf{x}^{(i)}\right)\right)\right)}, \quad \forall i \in \mathcal{M}_{n} \\
P\left(\mathbf{x}^{(i)}=\mathrm{NH}\left(\mathbf{x}^{(n)}\right) \mid \theta\right)=\frac{k\left(d\left(\mathbf{f}_{\theta}\left(\mathbf{x}^{(n)}\right), \mathbf{f}_{\theta}\left(\mathbf{x}^{(i)}\right)\right)\right)}{\sum_{j \in \mathcal{H}_{n}} k\left(d\left(\mathbf{f}_{\theta}\left(\mathbf{x}^{(n)}\right), \mathbf{f}_{\theta}\left(\mathbf{x}^{(i)}\right)\right)\right)}, \quad \forall i \in \mathcal{H}_{n}
\end{gathered}
$$

$k(\cdot)$ is a kernel function. We use the exponent kernel $k(d)=\exp (-d / \sigma)$, where $\sigma$ is a hyper-parameter that determines the resolution at which the data is locally analyzed. Now that the margins are defined, the problem of learning the mapping $\mathbf{f}_{\theta}$ can be solved within a large margin framework. The two most common margin 


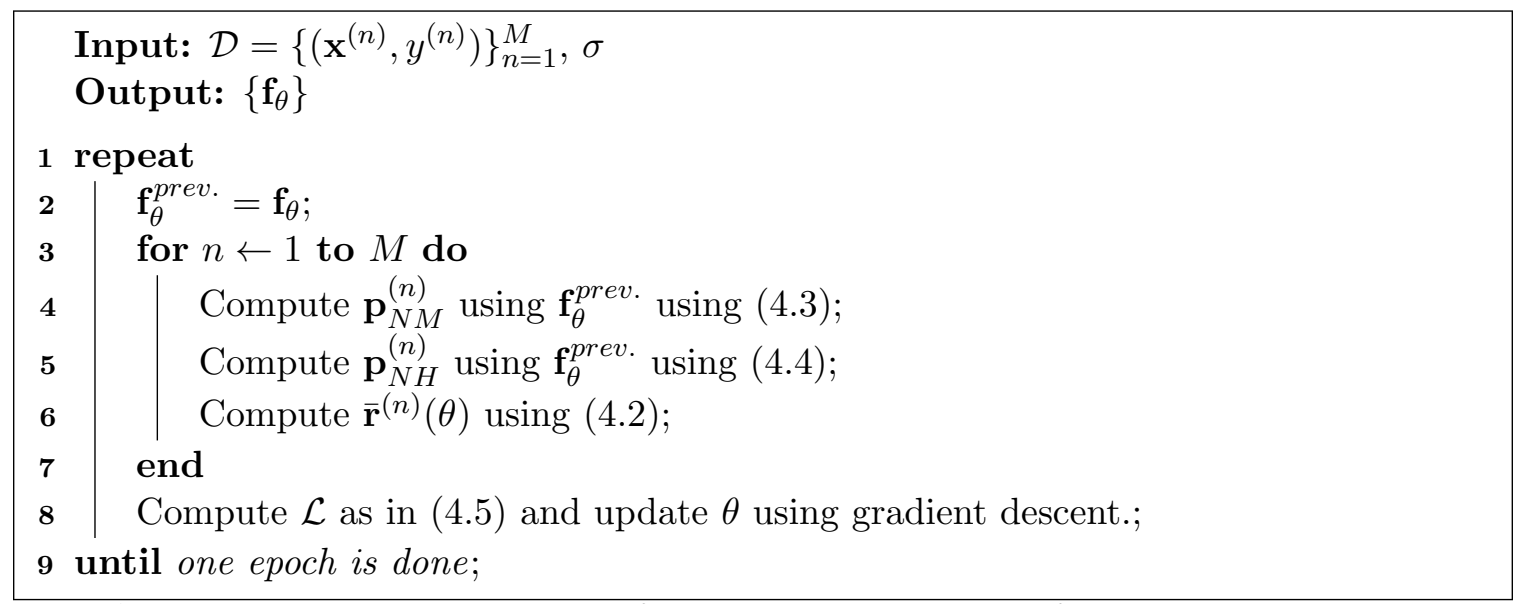

Algorithm 1: Pseudo code of the proposed method for one epoch.

formulations are based on Hinge loss and logistic regression. We use logistic regression, though experimentally we noticed similar results with Hinge loss as well. Using a logistic regression formulation leads to the following loss function. Pseudocode of the proposed method is shown in Algorithm 1.

$$
\mathcal{L}(\theta)=\sum_{n=1}^{M} \log \left(1+\exp \left(-\overline{\mathbf{r}}^{(n)}(\theta)\right)\right)
$$

Since the logistic loss function is an upper bound of the misclassification loss function, up to a difference of a constant factor, our algorithm can be regarded as minimizing the upper bound of the LOO classification error in the embedding space.

When $\sigma \rightarrow 0$, MsDNN will reduce to a triplet selection method where anchors simply pick their nearest positive and nearest negative samples to form triplets. Our experiments indeed confirmed that such an approach often does not converge. A similar issue is also reported for the popular triplet loss. While the mainstream approach to address this issue is based on carefully designing a strategy for triplet selection [3] or constructing batches [26], we suggest that this issue may be alleviated by replacing the positive and negative samples with their expected values. We will empirically validate this in the next chapter. 


\subsection{Summary}

In this chapter, we presented Multi-scale Deep Nearest Neighbor loss (MsDNN) for learning an embedding space. It builds on a probabilistic definition of sample margin. A sample is correctly classified if its margin is positive. MsDNN loss is differentiable. During training, MsDNN minimizes the upper bound of leave-one-out (LOO) 1-NN classification error. The output of MsDNN is an embedding space where classification can be done by for example k-NN classifier. An important characteristic of MsDNN is that it allows classes to have disjoint sets also known as subclasses or clusters. In the next chapter we will present the experiments designed to further evaluate the resulting embedding space. 


\section{Chapter 5}

\section{Experimental Results}

In this section, we demonstrate the ability of the MsDNN loss in learning similarities among samples at different scales. We demonstrate the effectiveness of the proposed method on one synthetic and four real-world datasets.

\subsection{Datasets}

The experiments are performed on 4 images based and 1 text-based dataset.

\subsubsection{MNIST}

The MNIST [49] (Modified National Institute of Standards and Technology database) is a dataset of handwritten digits that is comprised of 60,000 training samples and 10,000 test samples. Figure 5.1 shows some examples from MNIST dataset. The handwritten digits are of size $28 \times 28$ and don't need much preprocessing and formatting before use. This dataset is popular and thus many methods perform training and report results on MNIST. 

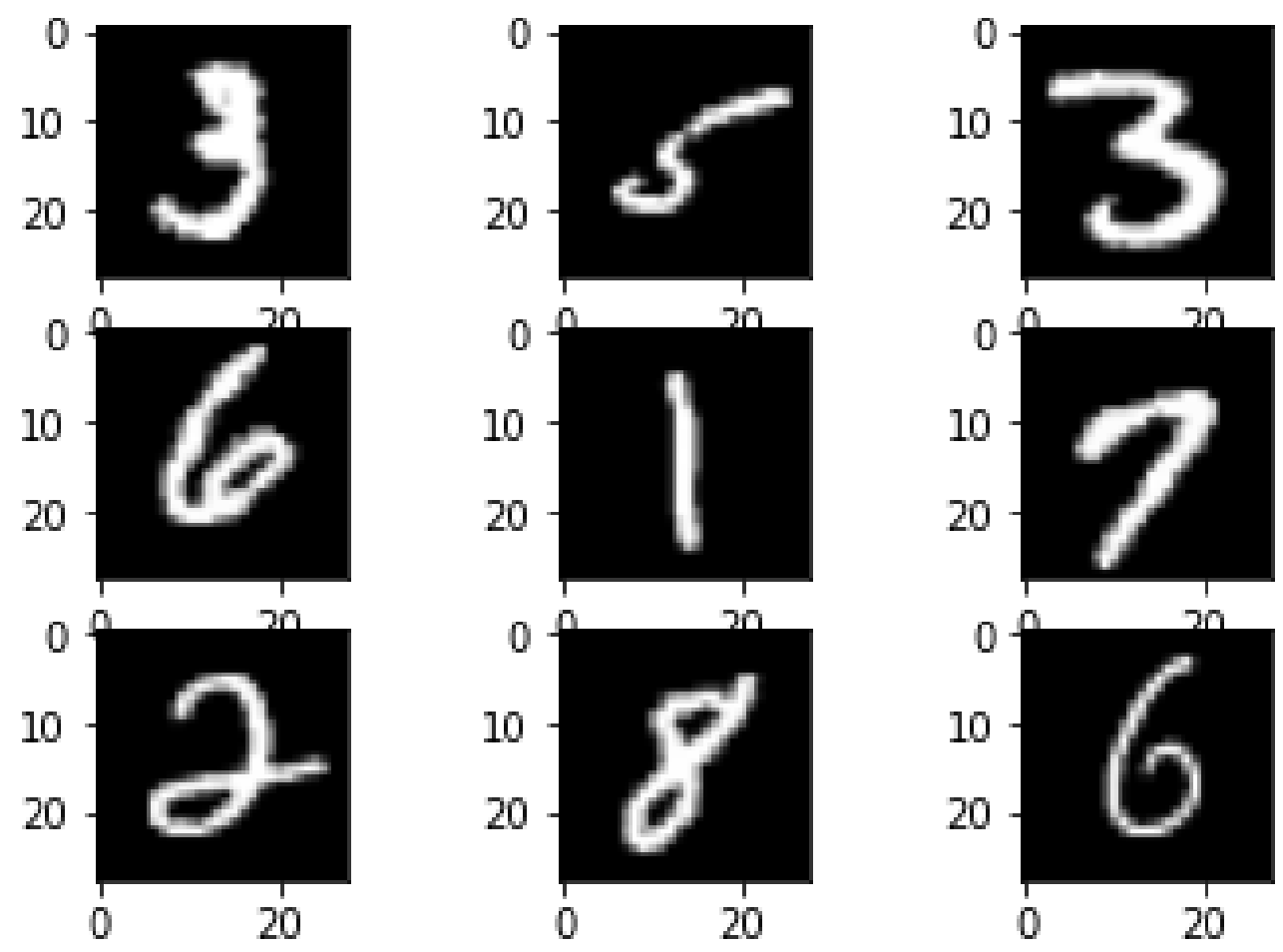

Figure 5.1: Examples from the MNIST dataset.

\subsubsection{Fashion MNIST}

The Fashion-MNIST [50] dataset is similar to MNIST because it also has 60,000 training examples and 10,000 test examples where each example is 28x28 grayscale images. Furthermore, both datasets have 10 classes. Fashion-MNIST can be easily used in place of MNIST. Fashion-MNIST is more complex as is evident from samples shown in Figure 5.2.

The 10 classes of the dataset are T-shirt/top, Trouser, Pullover, Dress, Coat, Sandal, Shirt, Sneaker, Bag, Ankle boot. These classes are much harder to learn compared to handwritten digits. 

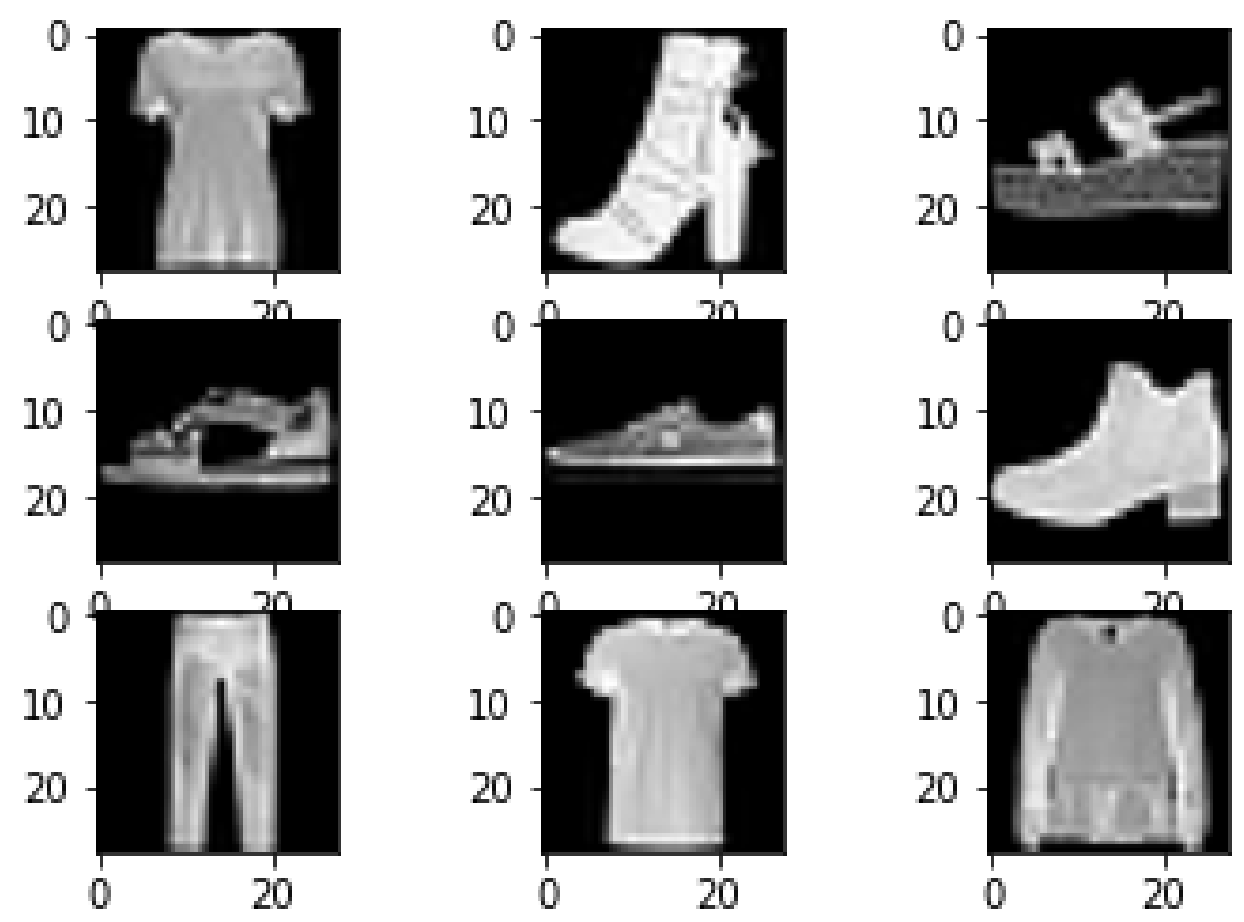

Figure 5.2: Examples from the FMNIST dataset.

\subsubsection{USPS}

The US Postal (USPS) [51] is a dataset of handwritten digit with 7291 train examples and 2007 test examples with each example of 16x16 grayscale images. This dataset is similar to MNIST in that both are handwritten digit as shown in Figure 5.3. Doing experiments on both may help to see how good a model generalize on similar data obtained using a different approach.

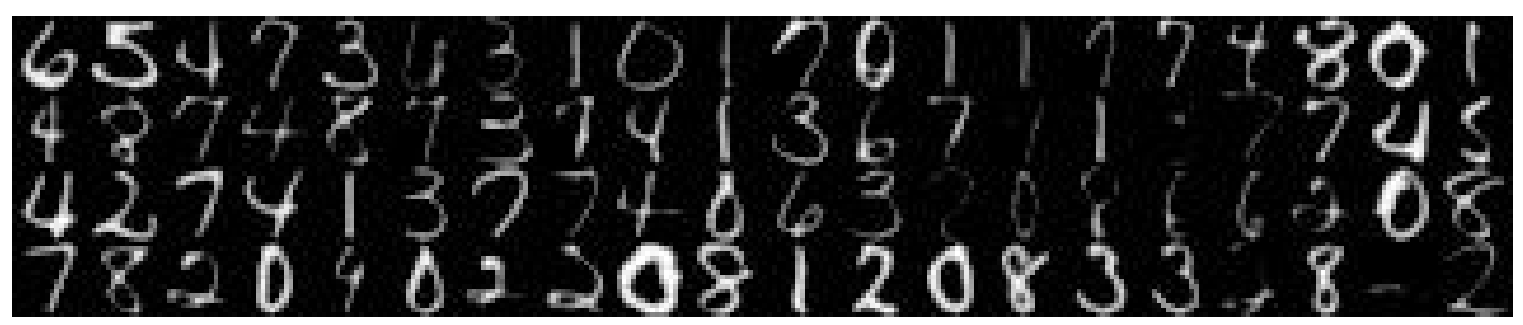

Figure 5.3: Examples from the USPS dataset [52]. 


\begin{tabular}{|c|c|c|}
\hline comp.graphics & rec.autos & sci.crypt \\
comp.os.ms-windows.misc & rec.motorcycles & sci.electronics \\
comp.sys.ibm.pc.hardware & rec.sport.baseball & sci.med \\
comp.sys.mac.hardware & rec.sport.hockey & sci.space \\
comp.windows.x & & \\
\hline misc.forsale & talk.politics.misc & talk.religion.misc \\
& talk.politics.guns & alt.atheism \\
& talk.politcs.mideast & soc.religion.christian \\
\hline
\end{tabular}

Table 5.1: 20 newsgroup topic grouped together based on the subject matter

\subsubsection{Newsgroup}

20 Newsgroup [53] dataset consists of 18000 newsgroup documents which are from 20 different topics. The train-test split is based on the documents posted before and after a specific date. On the one hand, some of these topics are related to each other. For example, rec.autos and rec.motorcycles are closely related to newsgroup topics. On the other hand, some of the topics are very different from each other. For example, misc.forsale and sci.space topics are highly unrelated to each other. The 20 classes grouped on the subject matter are shown in Table 5.1.

The articles in the dataset have headers including subject lines. One example of the article is :

Newsgroup: comp.os.ms-windows.misc

document_id: 10001

Subject: WIN STORM PC

From: srini@shannon.tisl.ukans.edu (Srini Seetharam)

Anyone have any info. on the video/sound card from SIGMA designs.

It is called WIN STORM PC.

They also have another card called the legend 24lx 
any info would be appreciated, including performance, pricing and availability.

thanks

srini

\subsection{Evaluation Metrics}

We evaluate the resulting space from two angles. From the classification view, during testing, we run the k-nn classifier and report the classification accuracy. But classification accuracy does not guarantee a good embedding space. Therefore, we run k-means clustering in the embedding space. We hypothesize that if a class consists of several disjoint sets (aka subclasses or clusters), a good embedding space should keep them separate. So, a simple clustering method such as k-means would identify them. We evaluate our embedding space for clustering and classification behavior. In the following, we describe the clustering measures that will be used to evaluate our method.

\subsubsection{Evaluating clustering performance}

To check the clustering performance of the embedding space learned, we use normalized mutual information (NMI) and clustering accuracy score for the clusters discovered using KMeans in the embedding space. 


\section{Clustering Accuracy}

After applying Kmeans, we get K clusters. Each data point in the dataset belongs to one cluster. Let $s_{i}$ be the learned cluster label and $r_{i}$ is the ground truth label. Then the clustering accuracy [54] is defined as:-

$$
\text { ClusteringAccuracy }=\frac{\sum_{i=1}^{N} I\left(s_{i}, \operatorname{map}\left(r_{i}\right)\right)}{N}
$$

where $\mathrm{N}$ is the total number of points, $\mathrm{I}(\mathrm{x}, \mathrm{y})$ function returns 1 when $\mathrm{x}=\mathrm{y}$ and it returns 0 when $x \neq y$. $\operatorname{map}\left(r_{i}\right.$ is a permutation mapping function that maps every learned cluster label to the ground truth label. Kuhn-Munkres algorithm can be used to find the best mapping.

\section{Normalized Mutual Information}

Normalized Mutual Information (NMI) [55] between two clusterings, i.e clusters from the ground truth labels and clusters obtained from the KMeans in the embedding space, is defined as the normalization of Mutual Information (MI). Consequently, NMI ranges from zero to one where zero means no mutual information, and 1 means perfect correlation. MI is normalized using the generalized mean of entropy of true labels $\left(H\left(L_{\text {true }}\right)\right)$ and entropy of predicted labels $H\left(L_{\text {pred }}\right)$. In our experiments, we use arithmetic means to normalize the MI. The equation to compute the NMI between two clusterings is defined as,

$$
\operatorname{NMI}\left(L_{\text {true }}, L_{\text {pred }}\right)=\frac{2 * M I\left(L_{\text {true }}, L_{\text {pred }}\right)}{H\left(L_{\text {true }}\right)+H\left(L_{\text {pred }}\right)}
$$

where MI is the mutual information and $\mathrm{H}$ is entropy. Entropy measures how uncertain the events are and is computed using Equation 5.3. It gives the average amount of information that is gained from sampling one sample from the input probability 
distribution.

$$
H(p)=-\sum_{i} p_{i} \log _{2}\left(p_{i}\right)
$$

Mutual Information informs us about the reduction in the entropy or uncertainty of true labels when we learn cluster labels. MI is high when the predicted labels reduce a lot of entropy of the true labels and it is low when there a very little reduction in the entropy of true labels. The MI is defined as,

$$
M I\left(L_{\text {true }}, L_{\text {pred }}\right)=H\left(L_{\text {true }}\right)-H\left(L_{\text {true }} \mid L_{\text {pred }}\right)
$$

where, $H\left(L_{\text {true }} \mid L_{\text {pred }}\right)$ is the conditional entropy of true labels given we have the predicted labels which can be computed using Equation 5.5.

$$
H(Y \mid X)=-\sum_{x \in X, y \in Y} p(x, y) \log _{2} \frac{p(x, y)}{p(x)}
$$

NMI is a popular evaluation metric for clustering. Different permutations of the predicted cluster label don't affect the score of NMI.

\subsubsection{Evaluating classification performance}

The classification accuracy is the fraction of correct predictions out of total predictions. Mathematically,

$$
\text { Classification Accuracy }=\frac{\text { Number of correct predictions }}{\text { Total number of predictions }}
$$




\subsection{MsDNN for learning at different scales}

We first present a simulation study on a synthesized dataset. This dataset consisted of 500 points in a 2-dimensional space where the points belong to two classes shown with different colors. The points in each subclass are sampled from a Gaussian distribution as shown in Figure 5.4 (a). We apply gradient descent to the dimensions of the input space. Through gradient descent, the points are moved in the input space such that the MsDNN loss be minimized. The parameter $\sigma$ controls the resolution at which samples are locally processed such that their margin is maximized. Figure 5.4 (b) shows the resulting input space when $\sigma$ is small. Likewise, Figure 5.4 (c) shows the resulting space when $\sigma$ is large. In both cases, the two classes become separated. When $\sigma$ is small, MsDNN preserves the subclasses. When $\sigma$ is large, subclasses merge. The proposed method not only learns to separate different classes but also enables us to look for subclasses at different scales.

To further illustrate the effect of $\sigma$ we carefully design experiments using the MNIST $^{1}$ dataset. MNIST originally has 10 classes. We define a binary classification problem on it: small numbers versus large numbers. Digits 0 to 4 belong to one class and digits 5 to 9 belong to the other class. This way, we simulate a binary classification problem where each class has five unknown subclasses. We trained a convolutional network on this dataset using MsDNN loss. See Section 5.6 for the network structure. Figure 5.5 shows the resulting embedding space for different values of $\sigma$ visualized using t-SNE [56]. Figure 5.5 (a) shows the t-SNE plots when $\sigma$ is small $\left(\right.$ here $\sigma=2^{-5}$ ). One may observe that the two classes are well separated and subclasses are also quite visible. For comparison, the ground-truth for the subclasses are shown in Figure 5.5 (b) using ten different colors. Figure 5.5 (c) shows the t-SNE plots when $\sigma$ is large (here $\sigma=1$ ). It can be seen that the two classes are well separated but no distinct

\footnotetext{
${ }^{1}$ http://yann.lecun.com/exdb/mnist/
} 


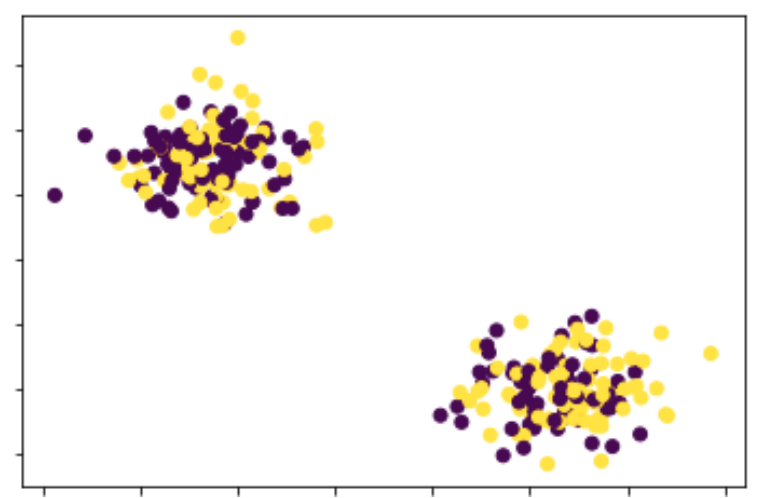

(a)

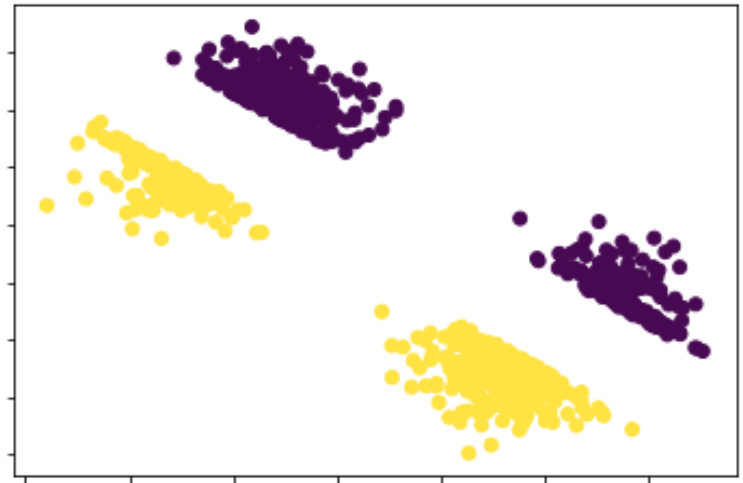

(b)

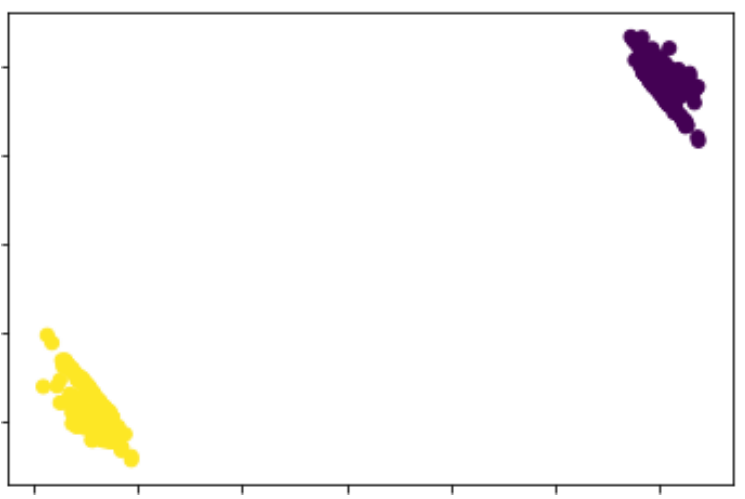

(c)

Figure 5.4: Effect of minimizing the MsDNN loss by applying gradient descent on the input space. (a) 2-dimensional synthetic data with two classes shown in yellow and purple. Each class initially has two subclasses. (b) The resulting input space when $\sigma$ is small. (c) The resulting input space when $\sigma$ is large.

subclass exists. The ground-truth for the subclasses are shown in Figure 5.5 (d) which confirms that the subclasses are blended.

Ideally, we would like to see that the distribution of samples from different subclasses overlap when $\sigma$ is large; and likewise, when $\sigma$ is small, subclasses come from distinct distributions. To verify this, the pair-wise symmetric Kullback-Leibler divergence between subclasses are shown in Figure 5.6. Figure 5.6 (a) shows the results when $\sigma$ is small, and Figure 5.6 (b) shows the results when $\sigma$ is large. For example, consider the yellow quarter at the top left corner of Figure 5.6 (b). This indicates that the symmetric KL divergence between the distribution of the subclasses are small 
which is in line with the t-SNE plot in Figure $5.5(\mathrm{~d})$. The network and the parameter $\sigma$ are the same as above. Moreover, we performed a similar experiment with USPS ${ }^{2}$ dataset and got similar results that are shown in Figure 5.6 (c) and (d).

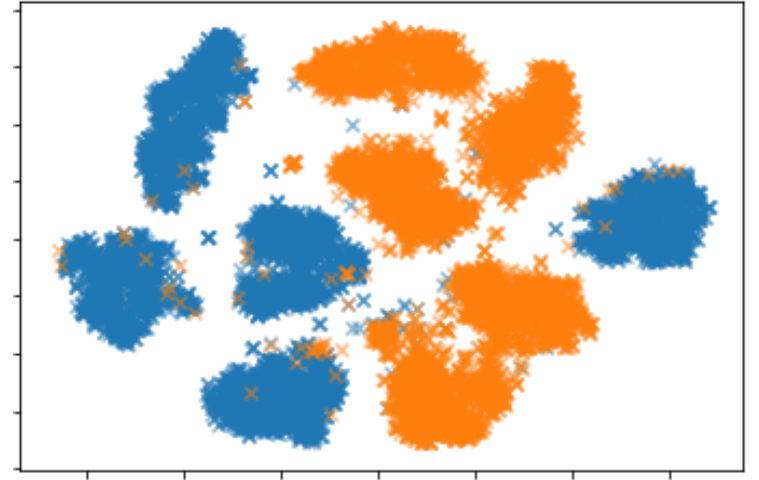

(a)

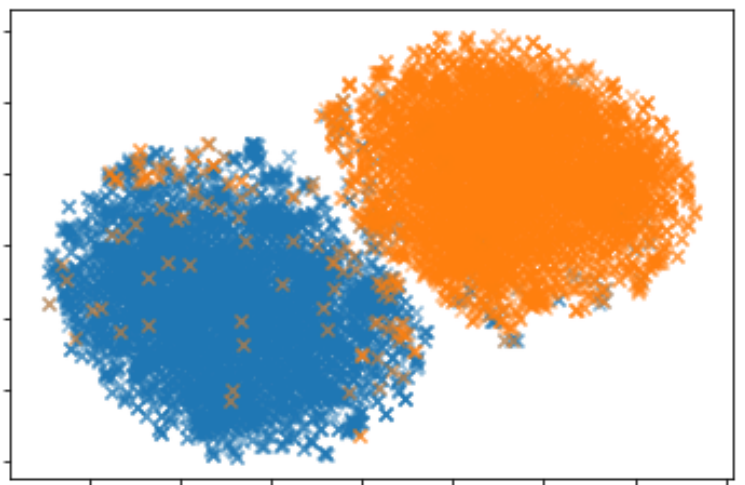

(c)

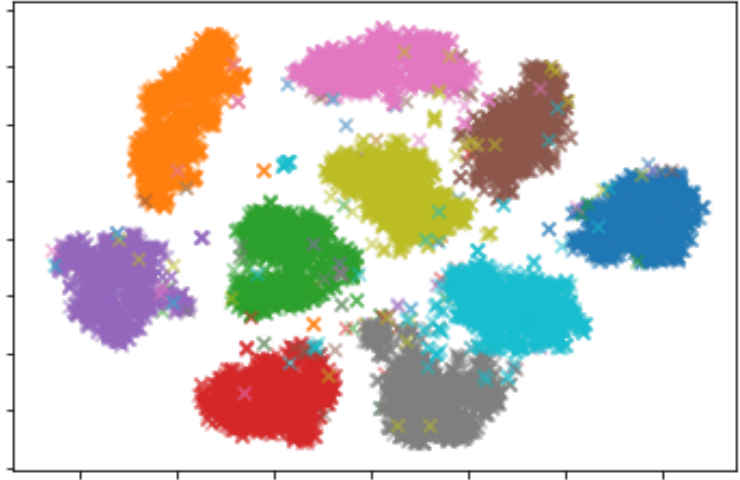

(b)

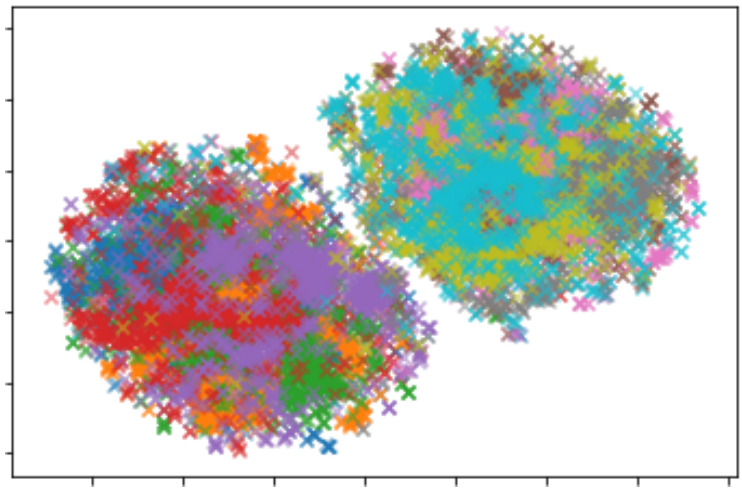

(d)

Figure 5.5: t-SNE plots for the MNIST dataset. (a) When $\sigma$ is small. Colors show the two classes. (b) The same as (a) but colors show the ground-truth values for the 10 subclasses. (c) When $\sigma$ is large. Colors show the two classes. (d) The same as (c) but colors show the ground-truth values the 10 subclasses.

We further investigate the performance of MsDNN for different values of $\sigma$ using Normalized Mutual Information (NMI). In addition to the MNIST and USPS, we perform experiments on 20NewsGroup ${ }^{3}$, and Fashion-MNIST ${ }^{4}$. All datasets except

\footnotetext{
${ }^{2}$ https://www.csie.ntu.edu.tw/ cjlin/libsvmtools/datasets/multiclass.html

${ }^{3}$ http://qwone.com/ jason/20Newsgroups/

${ }^{4}$ https://github.com/zalandoresearch/fashion-mnist
} 


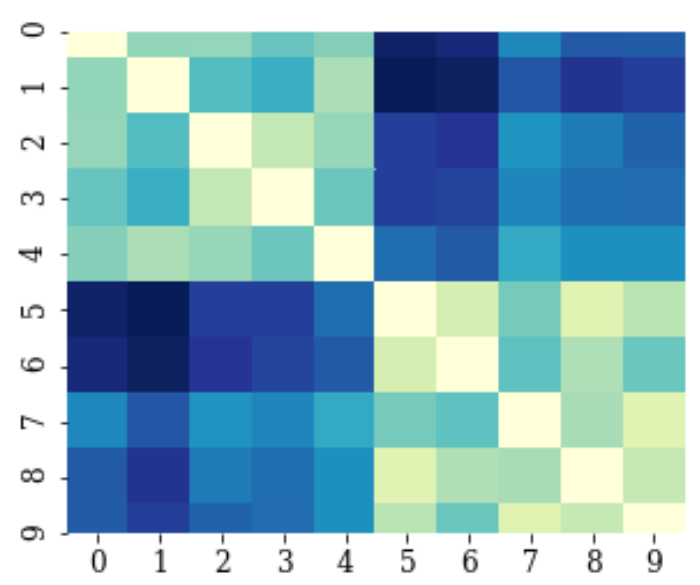

(a)

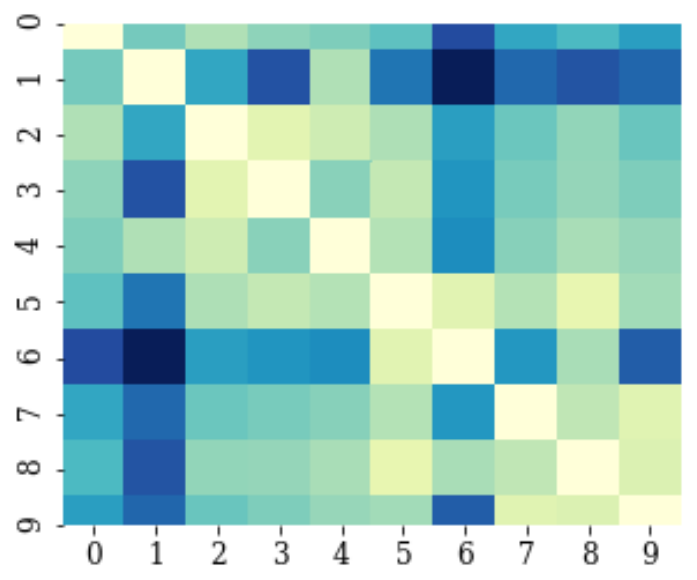

(c)

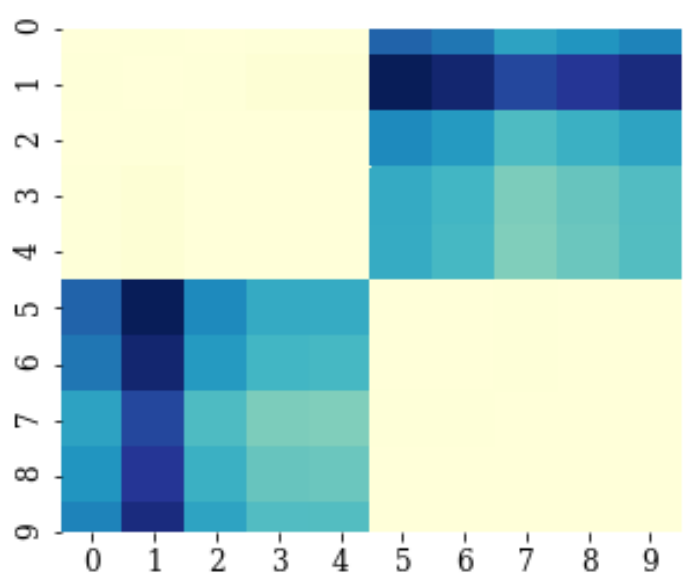

(b)

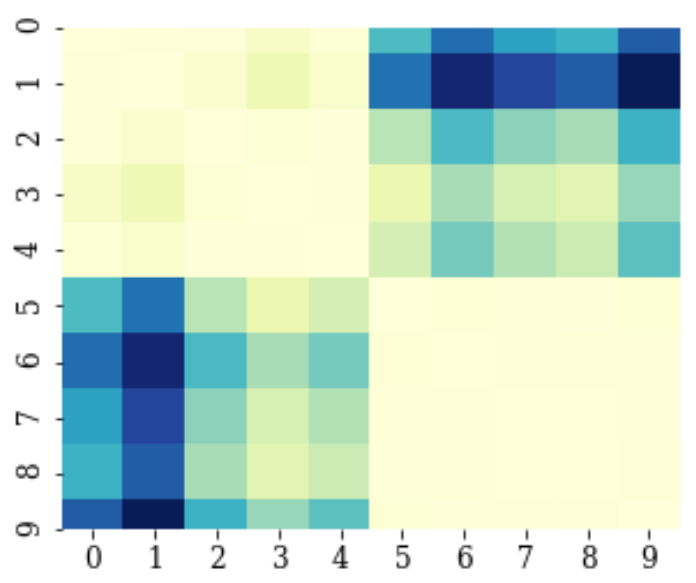

(d)

Figure 5.6: Pairwise symmetric KL-divergence between subclasses. (a) for the MNIST dataset when $\sigma$ is small. (b) for the MNIST dataset when $\sigma$ is large. (c) for the USPS dataset when $\sigma$ is small. (d) for the USPS dataset when $\sigma$ is large. Lighter colors denote lower KL-divergence.

the 20NewsGroup originally come with 10 classes and we simulate a binary classification problem for each dataset by assigning the first five classes to one class and the rest to another class. Each of the resulting datasets has two classes where each class contains five unknown subclasses. The 20NewsGroup dataset originally has twenty classes categorized into six categories. We pick the six categories as six classes where each class has several unknown subclasses. We train a classifier using MsDNN loss 


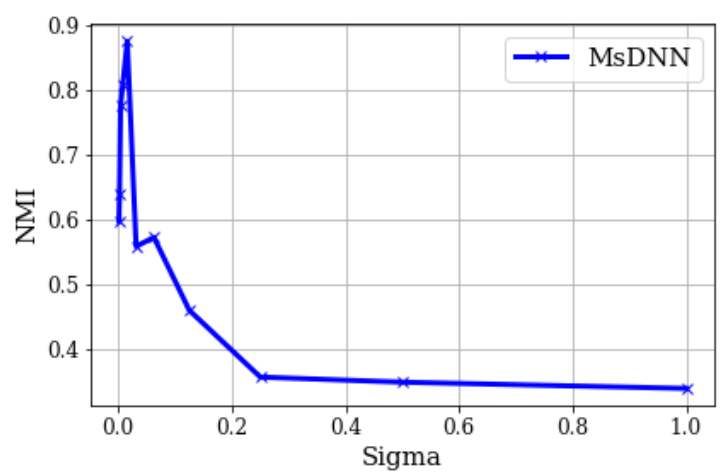

(a)

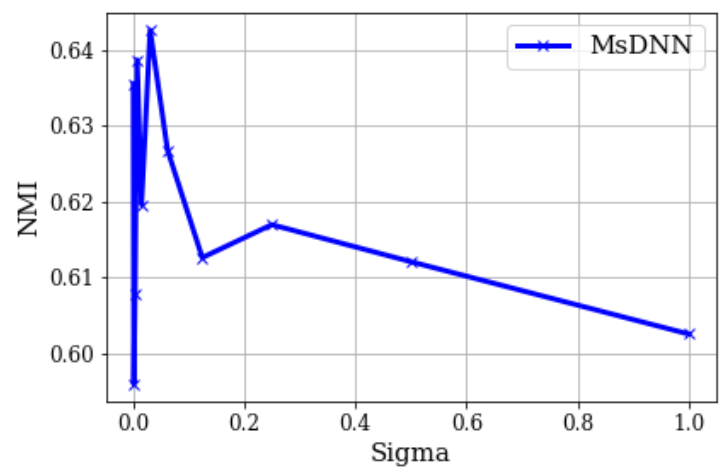

(c)

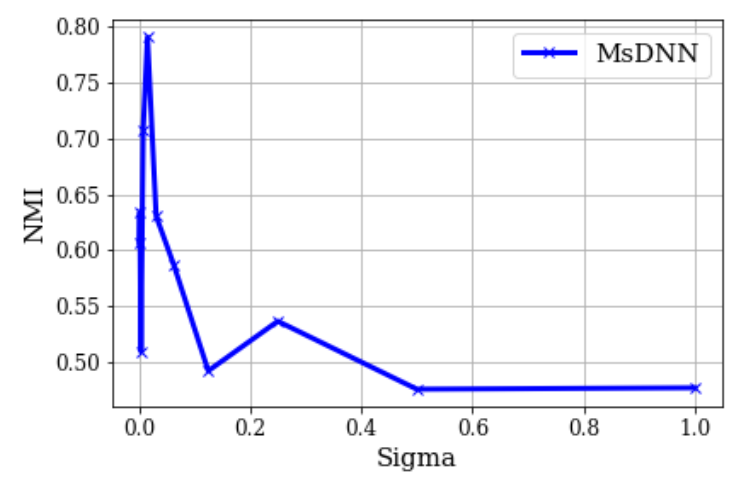

(b)

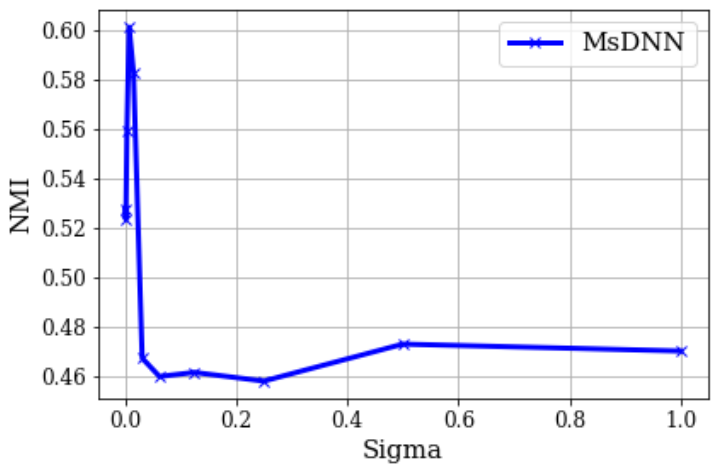

(d)

Figure 5.7: NMI for different values of $\sigma$. (a) MNIST. (b) USPS. (c) 20NewsGroup. (d) F-MNIST.

and run k-means in the resulting embedding space and compare the resulting clusters with the ground-truth using NMI. As shown in Figure 5.7, as $\sigma$ becomes larger, k-means' ability to detect the unknown subclasses in the embedding space diminishes, suggesting that the subclasses are being merged as the MsDNN processes data with a coarser resolution. Here, the parameter $\mathrm{k}$ in $\mathrm{k}$-means is set to the number of clusters/subclasses i.e. twenty for the 20NewsGroup and ten for the rest of the datasets. 


\subsection{Evaluating the embedding space}

In the following, we discuss the relation of the proposed MsDNN method to the broader category of metric learning methods and provide empirical evaluations. We compare the performance of the MsDNN with the soft nearest neighbor loss [46]. Note that other deep extensions of the k-NN including the neural nearest neighbor [44] and the deep k-NN [43] discussed in Section 3 do not provide a loss function and hence are not comparable with the proposed method. We also discuss the relation of the proposed MsDNN method to deep clustering methods and provide empirical evaluations.

Relation to metric learning methods: Metric learning methods have been used in many tasks such as face verification [57,58], image-based search [59], visual tracking $[60,61]$ and person re-identification $[62,63]$ where the goal is to learn a metric such that samples from the same class are more similar while samples from opposite classes are less similar. They treat each training class as a monolithic entity and usually lose most of the distinctions of the sub-divisions inside these classes. Most of the recent metric learning methods are based on deep learning, the two most common of which are Siamese networks and Triplet loss based networks [64]. Similar to MsDNN, the triplet loss [3] is based on a large margin framework applied to a set of triplets: anchor $p_{i}$, positive $p_{i}{ }^{+}$and negative $p_{i}{ }^{-}$. However, an important difference is that the triplet loss is based on the hardest positive and hardest negative samples. The choice of the hardest (farthest) positive instead of the nearest positive (nearest hit) effectively forces the subclasses to collapse into a single-mode. Moreover, the triplet loss is quite a discontinuous function and it is hard to train a neural network with it [3]. There has been a lot of works on devising strategies for triplet mining [65] and batch construction [26] to alleviate this issue. In contrast, MsDNN is seeking a different direction by making the margin function differentiable. Similarly, we modified the 
triplet loss by replacing the positive and negative samples with their expected values and run experiments on MNIST. The resulting loss converged and achieved about $99 \%$ classification accuracy for a wide range of $\sigma$. This is comparable to the performance of the triplet loss with hard negative mining method [3] but trains on average about $30 \%$ faster.

Relation to the deep clustering methods: Deep clustering methods learn an embedding space such that samples form tight clusters. MsDNN is a supervised method that learns an embedding space to distinguish different classes while allowing disjoint subclasses. Therefore, a shallow clustering method such as k-means may be used in the resulting embedding space to discover subclasses. Semi-supervised clustering methods are a category of clustering methods that use some auxiliary information, often in the form of cluster labels of a small portion of samples, to facilitate the clustering process. Since such data are not available during a classification task, we cannot compare our method with them. However, a subcategory of the semisupervised clustering methods is based on forming a set of pairwise constraints in the form of must-link and cannot-link pairs [70-75]. In a classification setting, we know that samples from opposite classes should not be in the same cluster. So, we can assume a cannot-link between them. But, the class labels provide no information about must-link pairs because a class might have distinct subclasses. Most of the existing deep semi-supervised clustering methods [73-77] requires both must-link and cannot-link information to function.

In general, a good embedding space should learn the similarity structures among samples. From a supervised learning perspective, classes should be well separated from each other. Moreover, beyond the class labels, the unknown subclasses should also be preserved. Here subclasses are the clusters within the classes. As a result, we evaluate our models from two different angles:

Clustering performance: We consider the k-means' ability in detecting subclasses 
Table 5.2: Evaluation of the proposed method on the train set. $\left(^{\dagger}\right),\left({ }^{\S}\right),\left(^{\ddagger}\right),\left({ }^{\dagger \dagger}\right)$ denotes that the results are reported in [66], [67], [68], and [69] respectively.

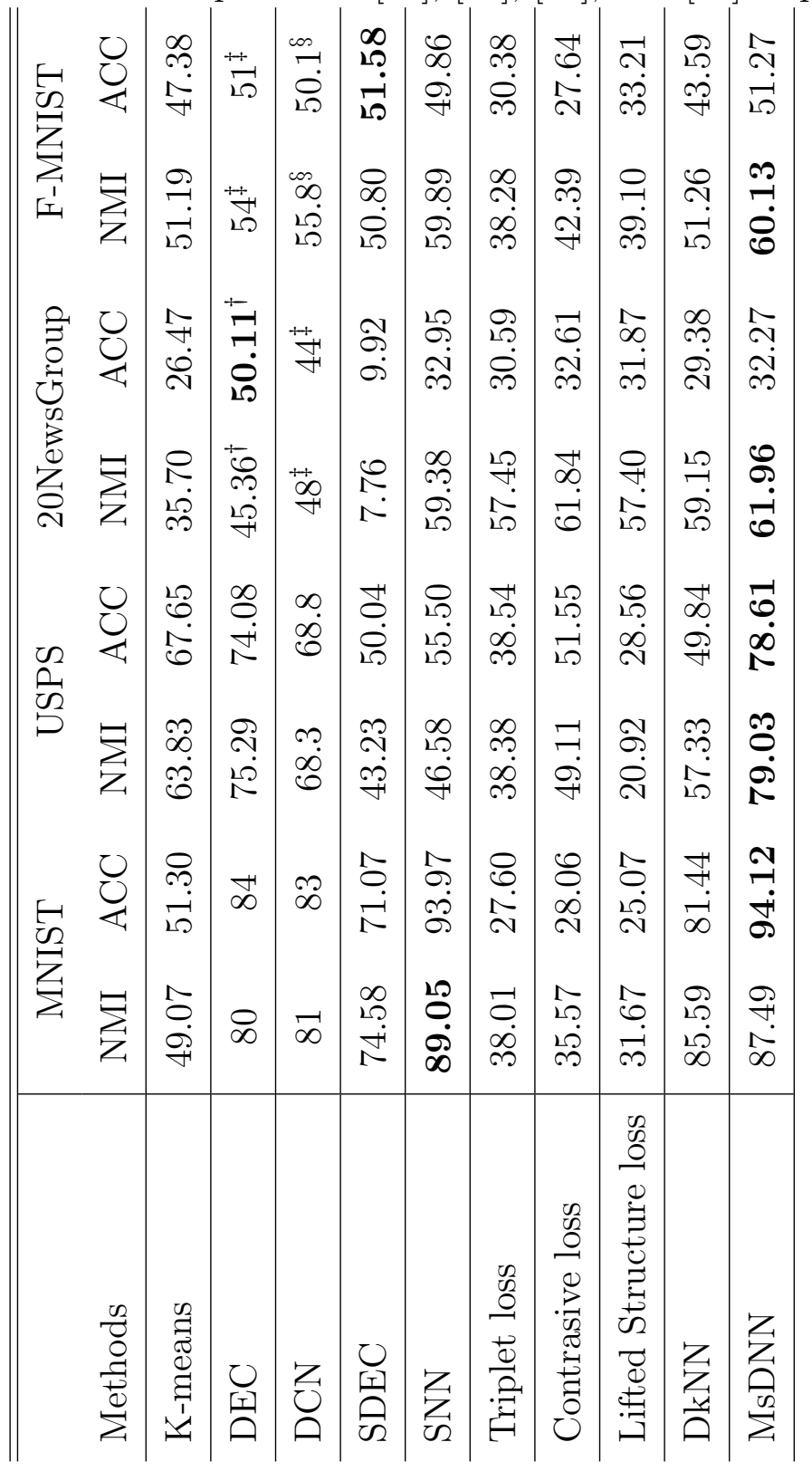


Table 5.3: Evaluation of the classification accuracy of the proposed method on the test set.

\begin{tabular}{l|ccccc}
\hline \hline & MNIST & USPS & 20NG & F-MNIST & AVG \\
\hline SNN & 99.19 & 85.95 & 90.56 & 94.64 & 92.59 \\
\hline Triplet & 99.24 & 95.15 & 87.47 & 94.80 & 94.17 \\
\hline Lifted Structure & 99.30 & 70.21 & 69.59 & 94.75 & 83.46 \\
\hline Contrastive & 99.04 & 92.09 & 88.72 & 94.03 & 93.47 \\
\hline MsDNN & 99.23 & 97.06 & 90.76 & 94.27 & $\mathbf{9 5 . 3 3}$ \\
\hline
\end{tabular}

as a measure of how well the subclasses are preserved in an embedding space. Table 5.2 shows the NMI and clustering accuracy of $\mathrm{k}$-means where the parameter $\mathrm{k}$ in k-means is set to the number of subclasses i.e. twenty for the 20NewsGroup and ten for the rest of the datasets.

Classification performance: We use a k-NN classifier to measure how well different classes are separated in the embedded space. Different values for $\mathrm{k}$ are tested from among 1, 3, 5, and 7 and the results on the test set for the best $\mathrm{k}$ are reported in Table 5.3. Note that this is a six-way classification for the 20NewsGroup and binary classification for the rest of the datasets.

In addition to the SNN loss, we compare the proposed methods with the metric learning methods discussed above including triplet loss [3], contrastive loss [78] and lifted structure loss [26]. Since we measure the clustering performance as a proxy to the true similarities between samples, the reader might be interested to know how clustering methods perform. Clustering methods do not use class labels but they are specialized in finding clusters. So, we also compare with the deep clustering methods including DEC [79], and DCN [80]. We also compare with SDEC which is a deep semi-supervised clustering method that can work in the absence of the must-link information. Though experimentally we observed that SDEC performs poorly in the absence of the must-link information. For reference, the performance of the k-means 
when applied to the input space is also reported.

It can be seen that the proposed methods on average outperform the comparison methods in both clustering and classification performance. These results confirm that in addition to learning to separate classes, the proposed methods learn to preserve the similarities between samples.

\subsection{Computational complexity}

For a batch of $M$ samples, MsDNN loss has $M$ margin terms and for each $2(M-1)$ distances should be computed. Therefore, following [81] the overall complexity of the MsDNN is $\mathrm{O}\left(n^{2}\right)$ where $n$ is the number of data points. This is better than the time complexity of the unmodified triplet loss which is $\mathrm{O}\left(n^{3}\right)$ [81]. In practice, we found that the training time of our method is similar to that of the triplet loss and SNN loss. For example, the average time per epoch on the MNIST dataset is 23,33 , and 21 seconds respectively for MsDNN loss, triplet loss, and SNN loss using a P100 GPU and four CPU cores. That is because the computational time for computing the gradient of the loss is negligible compared with the time needed to update the parameters of the backbone network. We run experiments for up to 100 epochs except for the USPS dataset where we run for 500 epochs.

\subsection{Implementation details}

We describe the implementation details about MsDNN in this section. The experiments were performed on an Intel E5-2650 v4 Broadwell CPU machine clocked at 2.2GHz. The experiments ran on four CPU cores with 32GB RAM and NVIDIA P100 Pascal GPU with 12G HBM2 memory. The storage was done in hard drive. We used the train/test splits that originally come with each dataset and MsDNN is 
implemented in Tensorflow 2.0.

\section{MNIST and Fashion MNIST}

MNIST and Fashion MNIST have the same dimension of images and both are grayscale. We use the same network architecture for them as demonstrated in Figure 5.8. This network is adapted from [82] For these datasets, we use a neural network with six convolutional layers followed by one fully connected layer. The kernel size of the convolutional layers is $(3,3)$ with the SAME padding and ReLU activation. The number of filters for the six layers are 2, 16, 32, 168, 256, and 32. And, it has a stride of $1,2,1,2,1$, and 1 starting from the input convolutional layer where the value of stride is equal in all directions. The output of the convolutional layers is flattened before it is fed to the fully connected layer which had nodes equal to the size of the embedding space. We learn an embedding size of 128 which is normalized with 12 normalization before being passed to MsDNN loss function. The network is optimized with an ADAM optimizer with a learning rate of 0.0001 and learned with a batch size of 1024 for 100 epochs.

\section{USPS}

For the USPS dataset, the network architecture, as shown in Figure 5.9, has six convolutional layers that are flattened before being passed to a fully connected layer with a number of nodes equal to the embedding size which is set to 32 for USPS. The output of this layer is 12 normalized. This architecture is similar to MNIST with the difference in the number of filters in convolutional layers. The number of filters for the six layers are $2,16,32,64,128$, and 32 . The network is optimized with an ADAM optimizer with a learning rate of 0.0001 and learned with a batch size of 1024 for 500 epochs. 


\begin{tabular}{|c|c|}
\hline 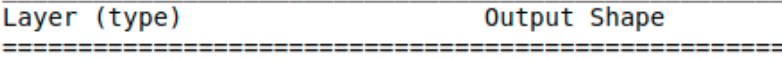 & 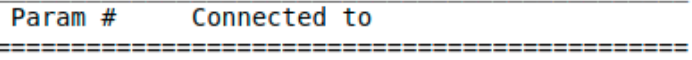 \\
\hline input_1 (InputLayer) $\quad[($ None, $28,28,1)]$ & $\theta$ \\
\hline (None, 28, 28, 2) & input_1[0][0] \\
\hline (None, 14, 14, 16) & conv2d $[0][0]$ \\
\hline (None, 14, 14, 32) & conv2d_1[0][0] \\
\hline (None, 7, 7, 128) & conv2d_2[0][0] \\
\hline (None, 7, 7, 256) & conv2d_3[0][0] \\
\hline conv2d_5 (Conv2D) & conv2d_4[0][0] \\
\hline flatten (Flatten) & conv2d_5[0][0] \\
\hline dense (Dense) & flatten $[\theta][\theta]$ \\
\hline tf_op_layer_l2_normalize/Square [(None, 128)] & dense $[\theta][\theta]$ \\
\hline tf_op_layer_l2_normalize/Sum (T [(None, 1)] & tf_op_layer_l2_normalize/Square $[\theta$ \\
\hline tf_op_layer_l2_normalize/Maximu [(None, 1)] & tf_op_layer_l2_normalize/Sum [0] [0 \\
\hline tf_op_layer_l2_normalize/Rsqrt [(None, 1)] & tf_op_layer_l2_normalize/Maximum [ \\
\hline 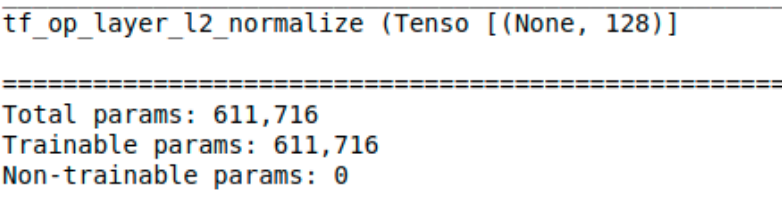 & 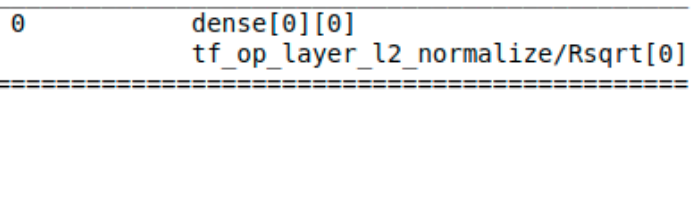 \\
\hline
\end{tabular}

Figure 5.8: Network Architecture for MNIST and Fashion MNIST.

\section{Newsgroup}

For the 20Newsgroup dataset, we used a deep feed-forward network. The input data was vectorized using tf-idf [83] with a maximum word count of 75000 before being fed to the neural network. The deep feed-forward network has 4 hidden layers with 512 nodes where each is followed with a Dropout layer with 0.5 being the probability of dropout. After 4 sets of hidden and dropout, data is passed through a fully connected layer with 100 nodes. Finally, the data is passed through another fully connected layer with several nodes equal to the embedding size which is set to 32 for this dataset. The output is 12 normalized before being passed to MsDNN loss. We optimize using the ADAM optimizer with a learning rate of 0.0001 . The network is trained for 100 epochs with a batch size of 4096 . 


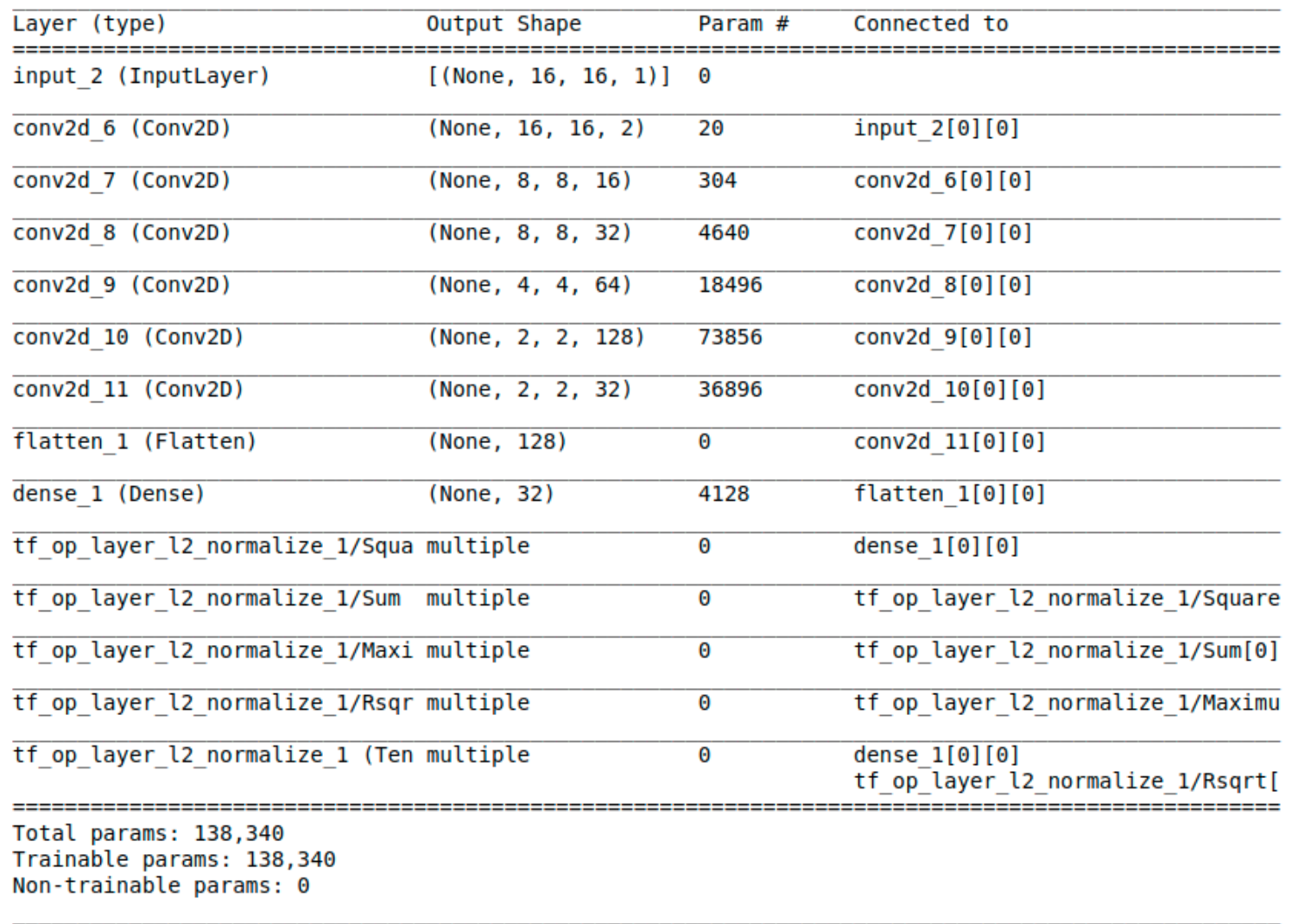

Figure 5.9: Network Architecture for USPS

\subsubsection{Model Selection}

The hyper-parameters $\sigma$ is searched among $\left\{2^{-10}, 2^{-9}, \cdots 2^{10}\right\}$. For the sake of fair comparison, for the temperature parameter of the SNN loss, we searched the same range as in $\sigma$ in MsDNN.

All datasets come with their standard train/test splits. For each dataset, we do an internal 5-fold cross-validation on the standard train set to select the best hyperparameters based on the classification accuracy. We then train on the entire standard train set using the selected hyperparameters and calculate classification accuracy on the test set. We want to highlight that for evaluating clustering performance, there is no such a notion of train/test splits. None of the comparison clustering methods have been considered a separate test set. This is a common practice in the clustering 


\begin{tabular}{|c|c|}
\hline 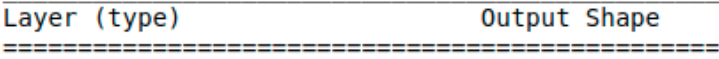 & 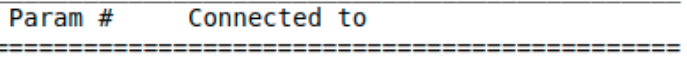 \\
\hline input_2 (InputLayer) [(None, 75000)] & $\theta$ \\
\hline dense_6 (Dense) & input_2[0][0] \\
\hline dropout_4 (Dropout) & dense_6[0][0] \\
\hline dense_7 (Dense) & dropout_4[0][0] \\
\hline dropout_5 (Dropout) & dense_7[0][0] \\
\hline dense_8 (Dense) & dropout_5[0][0] \\
\hline dropout_6 (Dropout) & dense_8[0][0] \\
\hline dense_9 (Dense) & dropout_6[0][0] \\
\hline dropout_7 (Dropout) & dense_9[0][0] \\
\hline dense_10 (Dense) & dropout_7[0][0] \\
\hline dense_11 (Dense) & dense_10[0][0] \\
\hline tf_op_layer_l2_normalize_1/Squa [(None, 32)] & dense_11[0][0] \\
\hline tf_op_layer_l2_normalize_1/Sum [(None, 1)] & tf_op_layer_l2_normalize_1/Square \\
\hline tf_op_layer_l2_normalize_1/Maxi [(None, 1)] & tf_op_layer_l2_normalize_1/Sum[0] \\
\hline tf_op_layer_l2_normalize_1/Rsqr [(None, 1)] & tf_op_layer_l2_normalize_1/Maximu \\
\hline 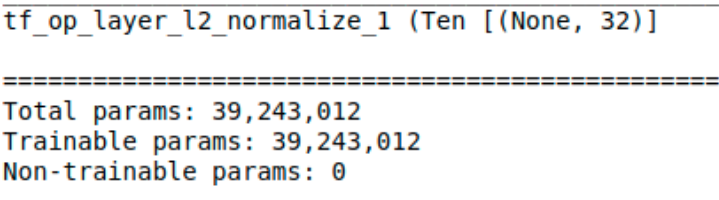 & $\begin{array}{ll}0 & \text { dense_11[0][0] } \\
& \text { tf_op_layer_l2_normalize_1/Rsqrt [ } \\
=======================================\end{array}$ \\
\hline
\end{tabular}

Figure 5.10: Network Architecture for 20Newsgroup

community [79]. Nevertheless, we want to highlight that MsDNN is not a clustering method, and we do not tune for clustering performance. We do model selection based on classification performance via cross-validation.

\subsection{Limitations}

MsDNN loss is minimized to learn a low dimensional embedding where the classes are separated and subclasses can be merged or distinct based on the value of $\sigma$. If the subclasses have the same size then one value of sigma can be used to find them. However, when the size of subclasses are different such that one subclass has few 
samples and another subclass has a lot of samples. In this case, there is no single value of $\sigma$ to discover them.

\subsection{Summary}

In our experiments, we demonstrate the effect of change in sigma to learn representations at different scales. When the sigma is small, we have clusters within the classes and when the sigma is large, the sub-classes merge to a single cluster for a class. The embedding space learned by MsDNN is evaluated for classification and clustering accuracy. Our method, on average has better clustering performance and classification accuracy. During experiments, the training time of MsDNN was found to be is similar to triplet loss and SNN loss. 


\section{Chapter 6}

\section{Conclusions}

In this thesis, we proposed a method for learning a deep embedding space suitable for k-NN. Our approach is based on minimizing the leave-one-out 1-NN classification error in the embedding space. That is, if we hold out a training sample, it should be correctly classified using 1-NN applied to the rest of the training data. Directly optimizing for such a rule is not tractable due to its discontinuous nature. We proposed Multi-scale Deep Nearest Neighbor (MsDNN) which is a differentiable loss function that is designed to maximize the expected sample margin for every training sample.

We evaluated the resulting space from two angles. From the classification view, during testing, we run a k-NN classifier and reported the classification accuracy. To further examine the quality of the resulting embedding space, we run k-means clustering in the embedding space to find subclasses that might exist inside the original classes. Analogous to the hierarchical clustering, subclasses might exist on different scales. Our method provides a mechanism to target subclasses in different scales. We empirically demonstrated that the embedding space learned by MsDNN can preserve the relationship between the data to discover subclasses while separating classes that were originally given during training.

We performed experiments on several datasets including four real-world and one

toy example. The method was compared with related work in metric learning and 
clustering and evaluated for both clustering and classification performance. Our method on average has the highest clustering performance while having comparable classification performance. Furthermore, we showed the effect of change in the scale parameter $\sigma$ to learn representation at different scales. When the value of $\sigma$ is low, we discover more subclasses, and as we increase sigma the number of subclasses decreases. 


\section{Chapter 7}

\section{Future Works}

A direction for further research could be to examine the application of the MsDNN loss in detecting adversarial attacks [84]. Adversarial examples are made by adding small perturbation to image from one class to make the prediction incorrect. Since our method retains the relationships between examples such that nearby examples in the input space are nearby in embedding space, it may provide robustness to adversarial attacks.

Further, MsDNN can be used to find subclasses in applications such as diagnosing autism spectrum disorder (ASD) or depression, where a large variability has been observed among the patients [85]. It has been suggested that such variability can be associated with the existence of some unknown homogeneous subgroups [85-87]. Identifying homogeneous subgroups is important because it may enable researchers to focus on each subgroup and find more efficient and effective treatments specific to that subgroup [87].

Since $\mathrm{MsDNN}$ is a combination of $\mathrm{k}-\mathrm{NN}$ and deep metric learning to learn an embedding space to improve the performance of the k-NN classifier, in the future, MsDNN can be applied to numerous applications where k-NN has been applied. Some of these areas of application are computer vision, natural language processing, healthcare, and recommendation systems. 


\section{List of References}

[1] O. Kramer, K-Nearest Neighbors, ch. 2, pp. 13-23. Berlin, Heidelberg: Springer Berlin Heidelberg, 2013.

[2] C. C. Aggarwal, A. Hinneburg, and D. A. Keim, "On the surprising behavior of distance metrics in high dimensional space," Lecture Notes in Computer Science (including subseries Lecture Notes in Artificial Intelligence and Lecture Notes in Bioinformatics), vol. 1973, pp. 420-434, 2001.

[3] F. Schroff, D. Kalenichenko, and J. Philbin, "Facenet: A unified embedding for face recognition and clustering," in Proceedings of the IEEE conference on computer vision and pattern recognition, pp. 815-823, 2015.

[4] T. George, S. P. Potty, and S. Jose, "Smile detection from still images using KNN algorithm," 2014 International Conference on Control, Instrumentation, Communication and Computational Technologies, ICCICCT 2014, pp. 461-465, 2014.

[5] S. S. Rautaray and A. Agrawal, "A novel human computer interface based on hand gesture recognition using computer vision techniques," ACM International Conference Proceeding Series, pp. 292-296, 2010.

[6] V. Bijalwan, V. Kumar, P. Kumari, and J. Pascual, "KNN based machine learning approach for text and document mining," International Journal of Database Theory and Application, vol. 7, no. 1, pp. 61-70, 2014.

[7] J. Vitola, F. Pozo, D. A. Tibaduiza, and M. Anaya, "A sensor data fusion system based on k-nearest neighbor pattern classification for structural health monitoring applications," Sensors (Switzerland), vol. 17, no. 2, 2017.

[8] J. Park and D. H. Lee, "Privacy preserving k-nearest neighbor for medical diagnosis in e-health cloud," Journal of Healthcare Engineering, vol. 2018, 2018. 
[9] R. Pan, P. Dolog, and G. Xu, "KNN-based clustering for improving social recommender systems," Lecture Notes in Computer Science (including subseries Lecture Notes in Artificial Intelligence and Lecture Notes in Bioinformatics), vol. 7607 LNAI, no. 247829, pp. 115-125, 2013.

[10] T. P. Salunke, "Recognition Based on Hog Feature Extraction And K-NN Classification," no. Iccmc, pp. 1151-1155, 2017.

[11] N. Dalal and B. Triggs, "Histograms of oriented gradients for human detection," Proceedings - 2005 IEEE Computer Society Conference on Computer Vision and Pattern Recognition, CVPR 2005, vol. I, pp. 886-893, 2005.

[12] M. Pietikäinen, A. Hadid, G. Zhao, and T. Ahonen, Computer vision using local binary patterns, vol. 40. Springer Science \& Business Media, 2011.

[13] A. Günay and V. V. NabIyev, "Automatic age classification with LBP," 2008 23rd International Symposium on Computer and Information Sciences, ISCIS 2008, pp. 6-9, 2008.

[14] B. Trstenjak, S. Mikac, and D. Donko, "KNN with TF-IDF based framework for text categorization," Procedia Engineering, vol. 69, pp. 1356-1364, 2014.

[15] J. Ramos et al., "Using tf-idf to determine word relevance in document queries," in Proceedings of the first instructional conference on machine learning, vol. 242, pp. 133-142, New Jersey, USA, 2003.

[16] J. Goldberger, S. Roweis, G. Hinton, and R. Salakhutdinov, "Neighbourhood components analysis," Advances in Neural Information Processing Systems, 2005.

[17] Weinberger, Kilian Q., John Blitzer and L. K. Saul., "Distance Metric Learning for Large Margin Nearest Neighbor Classification," Advances in Neural Information Processing Systems, pp. 1473-1480, 2006.

[18] K. Q. Weinberger and L. K. Saul, "Distance metric learning for large margin nearest neighbor classification," Journal of Machine Learning Research, vol. 10, pp. 207-244, 2009.

[19] I. Goodfellow, Y. Bengio, and A. Courville, Deep learning. MIT press, 2016.

[20] L. Yang and R. Jin, "Distance metric learning: A comprehensive survey," Michigan State Universiy, vol. 2, no. 2, p. 4, 2006. 
[21] "Dimensionality reduction by learning an invariant mapping," Proceedings of the IEEE Computer Society Conference on Computer Vision and Pattern Recognition, vol. 2, pp. 1735-1742, 2006.

[22] K. Q. Weinberger and L. K. Saul, "Distance metric learning for large margin nearest neighbor classification.," ournal of Machine Learning Research 10, no. 25, 2009.

[23] K. Sohn, "Improved deep metric learning with multi-class n-pair loss objective," in Advances in neural information processing systems, pp. 1857-1865, 2016.

[24] J. Wang, F. Zhou, S. Wen, X. Liu, and Y. Lin, "Deep Metric Learning with Angular Loss," Proceedings of the IEEE International Conference on Computer Vision, vol. 2017-October, pp. 2612-2620, 2017.

[25] L. Chen and Y. He, "Dress fashionably: Learn fashion collocation with deep mixed-category metric learning," 32nd AAAI Conference on Artificial Intelligence, AAAI 2018, pp. 2103-2110, 2018.

[26] H. Oh Song, Y. Xiang, S. Jegelka, and S. Savarese, "Deep metric learning via lifted structured feature embedding," in Proceedings of the IEEE conference on computer vision and pattern recognition, pp. 4004-4012, 2016.

[27] R. Hecht-Nielsen, "Theory of the backpropagation neural network," in Neural networks for perception, pp. 65-93, Elsevier, 1992.

[28] E. Alpaydin, Introduction to machine learning. MIT press, 2020.

[29] B. Kulis et al., "Metric learning: A survey," Foundations and trends in machine learning, vol. 5, no. 4, pp. 287-364, 2012.

[30] G. Cybenko, "Approximation by superpositions of a sigmoidal function," Mathematics of control, signals and systems, vol. 2, no. 4, pp. 303-314, 1989.

[31] B. Xu, N. Wang, T. Chen, and M. Li, "Empirical evaluation of rectified activations in convolutional network," arXiv preprint arXiv:1505.00853, 2015.

[32] D.-A. Clevert, T. Unterthiner, and S. Hochreiter, "Fast and accurate deep network learning by exponential linear units (elus)," arXiv preprint arXiv:1511.07289, 2015.

[33] T. P. Lillicrap, A. Santoro, L. Marris, C. J. Akerman, and G. Hinton, "Backpropagation and the brain," Nature Reviews Neuroscience, pp. 1-12, 2020. 
[34] N. Qian, "On the momentum term in gradient descent learning algorithms," Neural networks, vol. 12, no. 1, pp. 145-151, 1999.

[35] J. Duchi, E. Hazan, and Y. Singer, "Adaptive subgradient methods for online learning and stochastic optimization.," Journal of machine learning research, vol. 12 , no. $7,2011$.

[36] G. Hinton, N. Srivastava, and K. Swersky, "Neural networks for machine learning lecture 6a overview of mini-batch gradient descent," Cited on, vol. 14, no. 8, 2012.

[37] D. P. Kingma and J. Ba, "Adam: A method for stochastic optimization," arXiv preprint arXiv:1412.6980, 2014.

[38] Y. LeCun, Y. Bengio, et al., "Convolutional networks for images, speech, and time series," The handbook of brain theory and neural networks, vol. 3361, no. 10, p. 1995, 1995.

[39] Y. LeCun, B. E. Boser, J. S. Denker, D. Henderson, R. E. Howard, W. E. Hubbard, and L. D. Jackel, "Handwritten digit recognition with a back-propagation network," in Advances in neural information processing systems, pp. 396-404, 1990.

[40] A. Krizhevsky, I. Sutskever, and G. E. Hinton, "Imagenet classification with deep convolutional neural networks," in Advances in neural information processing systems, pp. 1097-1105, 2012.

[41] K. Simonyan and A. Zisserman, "Very deep convolutional networks for large-scale image recognition," arXiv preprint arXiv:1409.1556, 2014.

[42] K. He, X. Zhang, S. Ren, and J. Sun, "Deep residual learning for image recognition," in Proceedings of the IEEE conference on computer vision and pattern recognition, pp. 770-778, 2016.

[43] N. Papernot and P. McDaniel, "Deep k-nearest neighbors: Towards confident, interpretable and robust deep learning," arXiv preprint arXiv:1803.04765, 2018.

[44] T. Plötz and S. Roth, "Neural nearest neighbors networks," in Advances in Neural Information Processing Systems, pp. 1087-1098, 2018.

[45] R. Salakhutdinov and G. Hinton, "Learning a nonlinear embedding by preserving class neighbourhood structure," in Artificial Intelligence and Statistics, pp. 412419, 2007. 
[46] N. Frosst, N. Papernot, and G. Hinton, "Analyzing and improving representations with the soft nearest neighbor loss," arXiv preprint arXiv:1902.01889, 2019.

[47] W. F. C. Z. R. H. L. W. Guo, Qing and S. Wang., "Learning dynamic siamese network for visual object tracking.," in IEEE International Conference on Computer Vision, pp. 1763-1771, 2017.

[48] Y. Sun, S. Todorovic, and S. Goodison, "Local-learning-based feature selection for high-dimensional data analysis," IEEE transactions on pattern analysis and machine intelligence, vol. 32, no. 9, pp. 1610-1626, 2009.

[49] Y. LeCun, "The mnist database of handwritten digits," http://yann. lecun. com/exdb/mnist/, 1998.

[50] H. Xiao, K. Rasul, and R. Vollgraf, "Fashion-mnist: a novel image dataset for benchmarking machine learning algorithms," arXiv preprint arXiv:1708.07747, 2017.

[51] J. J. Hull, "A database for handwritten text recognition research," IEEE Transactions on pattern analysis and machine intelligence, vol. 16, no. 5, pp. 550-554, 1994.

[52] Y. Yang, Y. Hu, and F. Wu, "Sparse and low-rank subspace data clustering with manifold regularization learned by Local Linear Embedding," Applied Sciences (Switzerland), vol. 8, no. 11, 2018.

[53] K. Lang, "Newsweeder: Learning to filter netnews," in Proceedings of the Twelfth International Conference on Machine Learning, pp. 331-339, 1995.

[54] D. Cai, X. He, and J. Han, "Locally consistent concept factorization for document clustering," IEEE Transactions on Knowledge and Data Engineering, vol. 23, no. 6, pp. 902-913, 2010.

[55] C. Manning, P. Raghavan, and H. Schütze, "Introduction to information retrieval," Natural Language Engineering, vol. 16, no. 1, pp. 100-103, 2010.

[56] L. v. d. Maaten and G. Hinton, "Visualizing data using t-sne," Journal of machine learning research, vol. 9, no. Nov, pp. 2579-2605, 2008.

[57] J. Hu, J. Lu, and Y.-P. Tan, "Discriminative deep metric learning for face verification in the wild," in Proceedings of the IEEE conference on computer vision and pattern recognition, pp. 1875-1882, 2014. 
[58] Y. Sun, Y. Chen, X. Wang, and X. Tang, "Deep learning face representation by joint identification-verification," in Advances in neural information processing systems, pp. 1988-1996, 2014.

[59] P. Wu, S. C. Hoi, H. Xia, P. Zhao, D. Wang, and C. Miao, "Online multimodal deep similarity learning with application to image retrieval," in Proceedings of the 21st ACM international conference on Multimedia, pp. 153-162, 2013.

[60] J. Hu, J. Lu, and Y.-P. Tan, "Deep metric learning for visual tracking," IEEE Transactions on Circuits and Systems for Video Technology, vol. 26, no. 11, pp. 2056-2068, 2015.

[61] H. Li, Y. Li, and F. Porikli, "Deeptrack: Learning discriminative feature representations online for robust visual tracking," IEEE Transactions on Image Processing, vol. 25, no. 4, pp. 1834-1848, 2015.

[62] D. Yi, Z. Lei, S. Liao, and S. Z. Li, "Deep metric learning for person reidentification," in 2014 22nd International Conference on Pattern Recognition, pp. 34-39, IEEE, 2014.

[63] S. Liao, Y. Hu, X. Zhu, and S. Z. Li, "Person re-identification by local maximal occurrence representation and metric learning," in Proceedings of the IEEE conference on computer vision and pattern recognition, pp. 2197-2206, 2015.

[64] J. Lu, J. Hu, and J. Zhou, "Deep metric learning for visual understanding: An overview of recent advances," IEEE Signal Processing Magazine, vol. 34, no. 6, pp. 76-84, 2017.

[65] J. Wang, Y. Song, T. Leung, C. Rosenberg, J. Wang, J. Philbin, B. Chen, and $\mathrm{Y} . \mathrm{Wu}$, "Learning fine-grained image similarity with deep ranking," in Proceedings of the IEEE Conference on Computer Vision and Pattern Recognition, pp. 1386-1393, 2014.

[66] Y. Ren, K. Hu, X. Dai, L. Pan, S. C. Hoi, and Z. Xu, "Semi-supervised deep embedded clustering," Neurocomputing, vol. 325, pp. 121-130, 2019.

[67] Q. Zhu and Z. Wang, "An image clustering auto-encoder based on predefined evenly-distributed class centroids and mmd distance," Neural Processing Letters, pp. 1-16, 2020.

[68] S. E. Chazan, S. Gannot, and J. Goldberger, "Deep clustering based on a mixture of autoencoders," in 2019 IEEE 29th International Workshop on Machine Learning for Signal Processing (MLSP), pp. 1-6, 2019. 
[69] X. Li, H. Yin, K. Zhou, and X. Zhou, "Semi-supervised clustering with deep metric learning and graph embedding," World Wide Web, pp. 1-18, 2019.

[70] S. Basu, A. Banerjee, and R. Mooney, "Semi-supervised clustering by seeding," in In Proceedings of 19th International Conference on Machine Learning (ICML2002, Citeseer, 2002.

[71] B. Kulis, S. Basu, I. Dhillon, and R. Mooney, "Semi-supervised graph clustering: a kernel approach," Machine learning, vol. 74, no. 1, pp. 1-22, 2009.

[72] W. Tang, H. Xiong, S. Zhong, and J. Wu, "Enhancing semi-supervised clustering: a feature projection perspective," in Proceedings of the 13th ACM SIGKDD international conference on Knowledge discovery and data mining, pp. 707-716, 2007.

[73] G. Chen, "Deep transductive semi-supervised maximum margin clustering," arXiv preprint arXiv:1501.0623\%, 2015.

[74] D. Ienco and R. G. Pensa, "Deep triplet-driven semi-supervised embedding clustering," in International Conference on Discovery Science, pp. 220-234, Springer, 2019.

[75] L. A. Vilhagra, E. R. Fernandes, and B. M. Nogueira, "Textcsn: a semisupervised approach for text clustering using pairwise constraints and convolutional siamese network," in Proceedings of the 35th Annual ACM Symposium on Applied Computing, pp. 1135-1142, 2020.

[76] M. Śmieja, E. Struski, and M. A. Figueiredo, "A classification-based approach to semi-supervised clustering with pairwise constraints," Neural Networks, 2020.

[77] A. Shukla, G. S. Cheema, and S. Anand, "Semi-supervised clustering with neural networks," arXiv preprint arXiv:1806.01547, 2018.

[78] G. Koch, R. Zemel, and R. Salakhutdinov, "Siamese neural networks for one-shot image recognition," in ICML deep learning workshop, vol. 2, Lille, 2015.

[79] J. Xie, R. Girshick, and A. Farhadi, "Unsupervised deep embedding for clustering analysis," in International conference on machine learning, pp. 478-487, 2016.

[80] B. Yang, X. Fu, N. D. Sidiropoulos, and M. Hong, "Towards k-means-friendly spaces: Simultaneous deep learning and clustering," in Proceedings of the 34th International Conference on Machine Learning-Volume 70, pp. 3861-3870, JMLR. org, 2017. 
[81] T.-T. Do, T. Tran, I. Reid, V. Kumar, T. Hoang, and G. Carneiro, "A theoretically sound upper bound on the triplet loss for improving the efficiency of deep distance metric learning," in Proceedings of the IEEE Conference on Computer Vision and Pattern Recognition, pp. 10404-10413, 2019.

[82] Y. LeCun, L. Bottou, Y. Bengio, and P. Haffner, "Gradient-based learning applied to document recognition," Proceedings of the IEEE, vol. 86, no. 11, pp. 2278-2324, 1998.

[83] G. Salton and M. J. McGill, "Introduction to modern information retrieval," 1986.

[84] I. J. Goodfellow, J. Shlens, and C. Szegedy, "Explaining and harnessing adversarial examples," arXiv preprint arXiv:1412.6572, 2014.

[85] R. K. Lenroot and P. K. Yeung, "Heterogeneity within autism spectrum disorders: what have we learned from neuroimaging studies?," Frontiers in human neuroscience, vol. 7, p. 733, 2013.

[86] H. Witt, S. C. Mack, M. Ryzhova, S. Bender, M. Sill, R. Isserlin, A. Benner, T. Hielscher, T. Milde, M. Remke, et al., "Delineation of two clinically and molecularly distinct subgroups of posterior fossa ependymoma," Cancer cell, vol. 20, no. 2, pp. 143-157, 2011.

[87] J. Ellegood, E. Anagnostou, B. Babineau, J. Crawley, L. Lin, M. Genestine, E. Dicicco-Bloom, J. Lai, J. Foster, O. Penagarikano, et al., "Clustering autism: using neuroanatomical differences in 26 mouse models to gain insight into the heterogeneity," Molecular psychiatry, vol. 20, no. 1, pp. 118-125, 2015. 